SISSA / 69/2002/EP

SLAC-PUB-9543

hep-ph/0211375

October 29, 2018

\title{
Bounds on the Coupling of the Majoron to Light Neutrinos from Supernova Cooling *
}

\author{
Yasaman Farzan \\ Stanford Linear Accelerator Center, 2575 Sand Hill Road, Menlo Park, CA 94025 \\ and \\ Scuola Internazionale superiore di Studi Avanzati, via Beirut 4, I-34014 Trieste, Italy
}

\begin{abstract}
We explore the role of Majoron $(J)$ emission in the supernova cooling process, as a source of the upper bound on neutrino-Majoron coupling. We show that the strongest upper bound on the coupling to $\nu_{e}$ comes from the $\nu_{e} \nu_{e} \rightarrow J$ process in the core of a supernova. We also find bounds on diagonal couplings of the Majoron to $\nu_{\mu(\tau)} \nu_{\mu(\tau)}$ and on off-diagonal $\nu_{e} \nu_{\mu(\tau)}$ couplings in various regions of the parameter space. We discuss the evaluation of the cross-section for four-particle interactions $(\nu \nu \rightarrow J J$ and $\nu J \rightarrow \nu J)$. We show that these are typically dominated by three-particle sub-processes and do not give new independent constraints.
\end{abstract}

\section{Introduction}

The solar and atmospheric neutrino observations provide strong evidence in favor of neutrinos being massive. These experiments are sensitive only to mass squared differences [1], on the

${ }^{*}$ Work supported, in part, by the U. S. Department of Energy under contract DE-AC03-76SF00515. 
other hand, the overall mass scale of neutrinos is strongly constrained by the Troitsk and Mainz experiments [2]. Combining these pieces of information, we conclude that the masses of the three active neutrinos are very small. Among the plausible and economic models which are developed to give a tiny mass to neutrinos are Majoron models [3, 4]. In these models, additional Higgs boson(s) are introduced such that their vacuum expectation values break the exact B-L symmetry of the model. The Goldstone boson associated with this symmetry breaking is called the Majoron particle, $J$.

In principle, Majoron particles can interact with matter -electrons, nuclei and photons. However the cooling of red giant stars provides a strong bound on these interactions [5]. Hereafter, we will assume that Majorons can interact only with neutrinos. In the literature, two types of Majoron interaction have been studied:

$$
\mathcal{L}_{\text {int }}=\frac{1}{2} J\left(g_{\alpha \beta} \Phi_{\alpha}^{T} \sigma_{2} \Phi_{\beta}+g_{\alpha \beta}^{*} \Phi_{\beta}^{\dagger} \sigma_{2} \Phi_{\alpha}^{*}\right)
$$

and

$$
\mathcal{L}_{i n t}=h_{\alpha \beta} \Phi_{\alpha}^{\dagger} \bar{\sigma} \cdot(\partial J) \Phi_{\beta}
$$

where $J$ is the Majoron field, $\Phi_{\beta}$ is a two-component representation of a neutrino of flavor $\beta, g_{\alpha \beta}$ and $h_{\alpha \beta}$ are $3 \times 3$ coupling matrices. The matrix $h_{\alpha \beta}$ is Hermitian while $g_{\alpha \beta}$ is a symmetric matrix. In the model [3], for a range of parameters, the interactions can be described by Eq. (1) (see the appendix of Ref. [6]). In this paper, we will use this form of the interaction however, as we will see later, in most cases our results apply for both forms. Also, we will not assume any special condition on the diagonal or off-diagonal elements of $g_{\alpha \beta}$. Since we have chosen a general approach, our results apply to any massless scalar field that has an interaction of the form given by Eq. (1), independent of the underlying model for it.

Majoron models are also interesting from the astrophysical point of view because, they provide the only mechanism for fast neutrino decay which has not yet been excluded ([7] and the references therein). The role of neutrino decay in the solar [8] and atmospheric [9] neutrino fluxes has been extensively studied. The possibility of explaining the anomalies by pure neutrino decay is excluded. In Ref. [10], decay of solar neutrinos along with oscillation has been discussed and, for a normal hierarchical neutrino mass scheme, it has been found that

$$
\left|g_{21}\right|^{2}=\left|\sum_{\alpha, \beta} g_{\alpha \beta} U_{\alpha 2}^{*} U_{\beta 1}\right|^{2} \leq 3 \times 10^{-5}\left(\frac{10^{-5} \mathrm{eV}^{2}}{\Delta m_{\text {sun }}^{2}}\right) .
$$

In Ref. [11], the different aspects and consequences of decay of neutrinos emitted by supernovae, have been studied. Future supernova observations can provide strong bounds 
on (or evidence for) neutrino decay and consequently on the Majoron coupling, provided that the uncertainties in supernova models are resolved.

If Majorons are coupled to neutrinos strongly enough, they can show up in $\beta \beta$-decay experiments, changing the spectrum of the final electrons. Non-observation of such an effect imposes a strong bound on the coupling constant [12]:

$$
\left|g_{e e}\right|<3 \times 10^{-5}
$$

Also, no sign of Majoron particles has been observed in the pion and kaon decays and therefore [13]:

$$
\sum_{l=e, \mu, \tau}\left|g_{e l}\right|^{2} \leq 3 \times 10^{-5} \quad \text { and } \quad \sum_{l=e, \mu, \tau}\left|g_{\mu l}\right|^{2} \leq 2.4 \times 10^{-4}
$$

The strongest bounds on neutrino-Majoron coupling are obtained by studying the role of these particles in a supernova explosion. In fact, three types of bounds are obtained:

i) Deleptonization: if the coupling, $\left|g_{e e}\right|$ is too large, Majoron emission can reduce the lepton number of the core of supernova via $\nu_{e} \rightarrow \bar{\nu}_{e} J$, preventing the emission of an intense observable photon flux. In $[14,15,16,17]$ this effect has been studied; the result is

$$
\left|g_{e e}\right|<2 \times 10^{-6}
$$

This bound strongly depends on the details of the supernova explosion model.

ii) Spectrum distortion: the production and absorption of the Majoron particle can affect the spectrum of the observed neutrino flux from a supernova explosion. This effect has been studied in Refs. [17, 15] and the result is

$$
\left|g_{11}\right|=\left|\sum_{\alpha, \beta} U_{\alpha 1}^{*} U_{\beta 1} g_{\alpha \beta}\right|<10^{-4}
$$

This result suffers from the low statistics of the SN1987a data and can be improved by future supernova observations.

iii) Energy loss: according to [18], the binding energy of a supernova core is $E_{b}=(1.5-$ $4.5) \times 10^{53} \mathrm{erg}$, which coincides very well with the energy emitted by SN1987a in 1-10 sec in the form of neutrinos. Hence the power carried away by any exotic particle such as Majoron cannot be larger than $\sim 10^{53} \mathrm{erg} / \mathrm{sec}$. This imposes strong bounds on the coupling of Majorons. The effect of energy transfer due to Majoron emission has been studied in a number of papers $[14,15,6,19,20,21]$.

In the presence of matter effects, a number of three-point processes that are kinematically forbidden in vacuum become allowed. For example, neutrino decay becomes possible even 
in the absence of neutrino masses. Also, neutrino annihilation into a massless Majoron, $\nu \nu \rightarrow J$, becomes kinematically allowed. The latter process has not been taken into account in previous studies. We will see that this is actually the dominant process contributing to energy loss in a supernova explosion. Previous studies must be reconsidered to take this effect into account.

In addition, the previous papers either considered only $g_{e e}$ or studied the Majoron couplings collectively without attention to the interplay of diagonal and off-diagonal couplings. In this paper, we study the effect of Majoron emission in the cooling process of supernova core considering all the relevant processes. We find that even for very small values of coupling, interplay of different processes may change the neutrino densities inside the supernova, evading the bounds that would be valid without this effect.

If the couplings are larger than some "lower" bounds, Majorons will be so strongly trapped inside the supernova that they cannot give rise to significant luminosity. Note that these "lower" bounds should be much larger than the limits at which Majorons start to become trapped. For such large values of coupling, Majoron production can completely change the density profile of the core by transferring energy between different layers and by changing lepton numbers. In this paper we discuss Majoron decay and all other processes that prevent energy transfer by Majoron particles and derive the limits on coupling constants above which the produced Majoron cannot leave the core without undergoing scattering or decay. We do not attempt to calculate any "lower" bound on the coupling constants, because for large values of couplings, the density distributions inside the core need to be recalculated. However we evaluate four-point processes which become important for large values of coupling constants. In summary, there is an "upper" bound on coupling below which the rate of Majoron production is so low that it cannot affect the evolution of supernovae. The values of coupling above the "upper" bound up to a "lower" bound are not allowed. However, the values of coupling above the "lower" bound (which are also higher than the "upper" bound) are not forbidden by supernova cooling considerations because for such values of coupling, Majorons cannot escape the core freely. The forbidden range is then between the "upper" and "lower" bounds. In figure (1), we illustrate all the bounds on $\left|g_{e e}\right|$ to clarify the meaning of the "upper" and "lower" bounds. The shaded area is excluded by the supernova cooling process.

This paper is organized as follows. In section 2, we calculate the cross-section of the relevant processes. In section 3, we briefly review the characteristics of the core. In section 4, we derive the bounds on the coupling constants and the values above which the produced 
Majoron will scatter before leaving the core. Conclusions are presented in section 5.

\section{Majoron interactions}

In this section we first introduce the Lagrangian. Then, in subsection 2.1, we derive the formulae for the neutrino propagator and the dispersion relation in the presence of matter. The interaction rates for different processes involving Majoron are derived in subsection 2.2.

In the presence of matter, the Lagrangian of neutrinos can be written in the twocomponent formalism as

$$
\mathcal{L}=\Phi_{\alpha}^{\dagger}\left(i \delta_{\alpha \beta} \bar{\sigma} . \partial-V_{\alpha \beta}\right) \Phi_{\beta}-\frac{m_{\alpha \beta}}{2}\left(\Phi_{\alpha}^{T} C \Phi_{\beta}-\Phi_{\beta}^{\dagger} C \Phi_{\alpha}^{*}\right)
$$

where $C=i \sigma_{2}, \alpha$ and $\beta$ are flavor indices, $\bar{\sigma}=(1,-\vec{\sigma})$ and $m_{\alpha \beta}$ is the symmetric Majorana mass matrix. The term $\Phi_{\alpha}^{\dagger} V_{\alpha \beta} \Phi_{\beta}$ represents the matter effect. This term has a preferred frame, the frame of the supernova. In the flavor basis, $\mathrm{V}$ is a diagonal matrix; $V=\operatorname{diag}\left(V_{e}, V_{\mu}, V_{\tau}\right)$ with

$$
V_{e}=V_{N}+V_{C}, \quad V_{\mu}=V_{\tau}=V_{N}
$$

where

$$
V_{C}=\sqrt{2} G_{F} n_{B}\left(Y_{e}+Y_{\nu_{e}}\right), \quad V_{N}=\sqrt{2} G_{F} n_{B}\left(-\frac{1}{2} Y_{n}+Y_{\nu_{e}}\right),
$$

$Y_{i}=\left(n_{i}-\bar{n}_{i}\right) / n_{B}$ and $n_{B}$ is the baryon density [22]. ${ }^{\dagger}$ In Eq. (5), the $Y_{\nu_{e}}$-dependent terms are the result of neutrino-neutrino scattering. Since in the medium of interest (supernova core) $n_{\nu_{\mu}}=n_{\bar{\nu}_{\mu}}$ and $n_{\nu_{\tau}}=n_{\bar{\nu}_{\tau}}{ }^{\ddagger}$ the corresponding $Y$ parameters vanish and have been omitted from Eq. (5). In Ref. [24] it is shown that due to loop effects the values of $V_{\mu}$ and $V_{\tau}$ are slightly different, however, the difference is negligible: $V_{\mu}-V_{\tau} \simeq 5 \times 10^{-5} V_{e}$ [25]. In a typical supernova core, $V_{\mu}$ and $V_{e}$ are of the order of $10 \mathrm{eV}$ and $1 \mathrm{eV}$, respectively.

\footnotetext{
${ }^{\dagger}$ It is shown in Ref. [23] that, if the neutrinos present in a medium are coherent superpositions of different flavor states, the off-diagonal elements of $V_{\alpha \beta}$ can be nonzero. However inside the inner core the densities of $\nu_{e}$ and $\nu_{\mu}$ are different and the densities of $\nu_{\mu}$ and $\nu_{\tau}$ are very low and equal to the densities of $\bar{\nu}_{\mu}$ and $\bar{\nu}_{\tau}$, so the off-diagonal terms vanish.

${ }^{\ddagger}$ In section 3, we will see that these equalities are only approximately true [30].
} 
For the interaction term, we invoke the form of Eq. (1). But we note that the derivative form of the interaction in Eq. (2) can be rewritten using the equations of motion as

$$
-i h_{\alpha \beta} m_{\beta \gamma} \Phi_{\alpha}^{\dagger} C \Phi_{\gamma}^{*} J-i h_{\alpha \beta} m_{\gamma \alpha} \Phi_{\gamma}^{T} C \Phi_{\beta}
$$

Thus, for processes in which all of the involved states are on-shell (in particular, neutrino and Majoron decay, $\nu \nu \rightarrow J$ and $\nu J \rightarrow \nu$ ) the two forms of interactions give the same results with the replacement

$$
g_{\alpha \beta} \rightarrow\left(h_{\alpha \gamma} m_{\gamma \beta}+m_{\alpha \gamma} h_{\gamma \beta}^{T}\right) .
$$

As we will see, the most important processes involve only on-shell particles. Therefore all of the bounds in this paper, apply for both derivative and pseudo-scalar forms of the interaction.

Majoron is a Goldstone boson associated with the exact B-L symmetry so in vacuum it is massless. Inside the supernova core Majoron obtains a tiny effective mass, $m_{e f f}$, due to elastic scattering off the background neutrinos. It can be shown that $m_{\text {eff }}^{2} \sim|g|^{2} N_{\nu} / q$ where $q$ is the typical momentum of the particles involved. For the values of coupling constants of order of the upper bounds the effective mass of Majoron is negligible $\left(m_{\text {eff }}^{2} / q \ll V_{e}\right)$. The effective mass can be considerable only if $|g| \gtrsim 5 \times 10^{-4}$.

\subsection{The propagators and the dispersion relation}

After straightforward calculations, we find

$$
\begin{gathered}
\sum_{\sigma, \gamma}\left[\left(\bar{\sigma} \cdot p-V_{\alpha}\right) \delta_{\alpha \sigma}-\frac{m_{\alpha \gamma} m_{\gamma \sigma}}{p \cdot \sigma+V_{\gamma}}\right]\left\langle\Phi_{\sigma}(p) \Phi_{\beta}^{\dagger}(-p)\right\rangle=i \delta_{\alpha \beta}, \\
\left\langle\Phi_{\alpha}^{*}(p) \Phi_{\beta}^{\dagger}(-p)\right\rangle=\sum_{\gamma} C \frac{m_{\alpha \gamma}}{p \cdot \sigma+V_{\alpha}}\left\langle\Phi_{\gamma}(p) \Phi_{\beta}^{\dagger}(-p)\right\rangle
\end{gathered}
$$

and

$$
\left\langle\Phi_{\alpha}(p) \Phi_{\beta}^{T}(-p)\right\rangle=\sum_{\gamma} m_{\beta \gamma}\left\langle\Phi_{\alpha}(p) \Phi_{\gamma}^{\dagger}(-p)\right\rangle \frac{-1}{p \cdot \sigma+V_{\beta}} C,
$$

where all of the subscripts $\alpha, \beta, \gamma$ and $\sigma$ denote $\{e, \mu, \tau\}$. As we will see, the diagrams in which these propagators are involved are important mainly when $|p| \lesssim V_{\alpha}$ so the effect of $V_{\alpha}$ must be treated non-perturbatively. If the mass scale of neutrinos is high $\left(m_{\nu} \gg \sqrt{\Delta m^{2}}\right)$, the masses are quasi-degenerate; $m_{\alpha \beta} \simeq m_{\nu} \delta_{\alpha \beta} \cdot{ }^{\S}$ In this case the formulae are simpler:

$$
\left\langle\Phi_{\alpha}(p) \Phi_{\beta}^{\dagger}(-p)\right\rangle=\frac{-i \delta_{\alpha \beta}}{m_{\nu}^{2}-\left(p \cdot \sigma+V_{\alpha}\right)\left(p \cdot \bar{\sigma}-V_{\alpha}\right)}\left(p \cdot \sigma+V_{\alpha}\right),
$$

\footnotetext{
$\S$ A proposed Tritium decay experiment, KATRIN [26], may be able to determine the mass scale.
} 


$$
\left\langle\Phi_{\alpha}^{*}(p) \Phi_{\beta}^{\dagger}(-p)\right\rangle=C \frac{-i m_{\nu} \delta_{\alpha \beta}}{m_{\nu}^{2}-\left(p \cdot \sigma+V_{\alpha}\right)\left(p \cdot \bar{\sigma}-V_{\alpha}\right)}
$$

and

$$
\left\langle\Phi_{\alpha}(p) \Phi_{\beta}^{T}(-p)\right\rangle=\frac{i m_{\nu} \delta_{\alpha \beta}}{m_{\nu}^{2}-\left(p \cdot \sigma+V_{\alpha}\right)\left(p \cdot \bar{\sigma}-V_{\alpha}\right)} C
$$

Now let us find the dispersion relation. The Lagrangian (3) yields

$$
\Phi_{\alpha}(p)^{\dagger}\left(-p \cdot \bar{\sigma}-V_{\alpha}\right)=\sum_{\beta} m_{\alpha \beta} \Phi_{\beta}^{T}(p) C
$$

and

$$
\left(p \cdot \bar{\sigma}-V_{\alpha}\right) \Phi_{\alpha}(p)=-\sum_{\beta} m_{\alpha \beta} C \Phi_{\beta}^{*}
$$

Expanding the states as

$\Phi_{\alpha}(p)=\sum_{h=-1,1} u_{\alpha}(h, p) a_{\alpha}(h, p)+v_{\alpha}^{\dagger}(h, p) a_{\alpha}^{\dagger}(h, p) \quad$ for $\quad$ which $\quad \vec{\sigma} \cdot \vec{p} u_{\alpha}(h, p)=h|\vec{p}| u_{\alpha}(h, p)$ we find

$$
\begin{gathered}
v_{\alpha}(h, p)=\sum_{\beta} m_{\alpha \beta} u_{\beta}^{T} \frac{C}{p^{0}-h|\vec{p}|+V_{\alpha}}, \\
u_{\alpha}(h, p)=\frac{-1}{p^{0}+h|\vec{p}|-V_{\alpha}} \sum_{\beta} m_{\alpha \beta} C v_{\beta}^{T}(h, p)
\end{gathered}
$$

and

$$
\left(p^{0}+h|\vec{p}|-V_{\alpha}\right) u_{\alpha}(h, p)=\sum_{\beta \gamma} \frac{m_{\alpha \beta} m_{\beta \gamma}}{\left(p^{0}-h|\vec{p}|+V_{\gamma}\right)} u_{\gamma}(h, p) .
$$

To find the dispersion relation and energy eigenstates we should solve Eq. (17). Note that the dispersion relation depends on helicity.

For $m^{2} / p \ll V \ll p$, one can easily show that

$$
p_{\alpha}^{0} \simeq p-h V_{\alpha}+\sum_{\beta} \frac{m_{\alpha \beta}^{2}}{2 p}
$$

and that the mixing among the flavors is of the order of $m^{2} / 2 p\left(V_{\beta}-V_{\alpha}\right) \ll 1$ which can be neglected.

\subsection{The relevant decays and interactions}

In this subsection we first discuss the processes that produce Majorons, then we study those that annihilate or scatter them. For illustrative reasons, in the following discussions, we 
ignore mixing (i.e., off-diagonal terms in both coupling and mass matrix) and we denote coupling, mass and effective potential by $g, m$ and $V$, neglecting their flavor indices. In the cases that generalization is not straightforward, we will discuss the relevant steps. Before beginning the detailed analysis, we should discuss an important conceptual point. As we see in Eq. (18) the dispersion relation for neutrinos inside supernova is different from that in vacuum and hence some reactions that are kinematically forbidden in vacuum, can take place in the supernova core. As we will see the decay $\bar{\nu} \rightarrow \nu+J$ and the interaction $\nu \nu \rightarrow J$ (or $\nu \rightarrow \bar{\nu} J$ and $\bar{\nu} \bar{\nu} \rightarrow J$ depending on the sign of $V$ ) are kinematically allowed.

In addition to these three-point interactions, there are other interactions that produce Majorons:

- $\nu+\nu \rightarrow J+J$ and $\bar{\nu}+\bar{\nu} \rightarrow J+J$

- $\nu+\bar{\nu} \rightarrow J+J$.

As we will see the effect of the four-point interactions is negligible.

\subsection{1 $\quad \bar{\nu} \rightarrow \nu+J$ or $\nu \rightarrow \bar{\nu}+J$}

In medium, if $V$ is negative (positive), the decay $\bar{\nu} \rightarrow \nu+J(\nu \rightarrow \bar{\nu}+J)$ is possible. Let us suppose $V<0$, then, without loss of generality, we can write

$$
p_{\bar{\nu}}=\left(p_{i}-V, 0,0, p_{i}\right) \quad p_{\nu}=\left(p_{f}+V, p_{f} \sin \theta, 0, p_{f} \cos \theta\right)
$$

where we have neglected corrections of order of $m^{2} / p_{i} \ll V$. Energy-momentum conservation implies that,

$$
p_{J}=\left(p_{i}-p_{f}-2 V,-p_{f} \sin \theta, 0, p_{i}-p_{f} \cos \theta\right) .
$$

Recalling that we have neglected the effective mass of Majoron, the process $\bar{\nu} \rightarrow \nu+J$ is kinematically allowed if and only if $p_{J}^{2}=0$ and all the zeroth components of the fourmomenta are positive. $p_{J}^{2}=0$ implies

$$
1-\cos \theta=\frac{4 V^{2}-2 V\left(p_{i}-p_{f}\right)}{2 p_{i} p_{f}} .
$$

For $|V|<p_{f}<p_{i}$, the above equation can be satisfied with all of the energies positive. This means that the process is kinematically allowed.

Restoring flavor indices, it can be shown that for $V_{\beta}+V_{\alpha}<0$ the rate of $\bar{\nu}_{\alpha} \rightarrow \nu_{\beta}+J$ is given by

$$
\frac{d \Gamma}{d p_{f}}=\frac{\left|g_{\alpha \beta}\right|^{2}}{8 \pi} \frac{p_{i}-p_{f}}{p_{i}^{2}}\left|V_{\alpha}+V_{\beta}\right| F_{\beta}^{F}\left(p_{f}\right)+\mathcal{O}\left(\frac{m^{2}}{p^{2}}\right)
$$


where $p_{i}$ and $p_{f}$ are the momenta of the initial and final neutrinos and $p_{f}$ extends from $\operatorname{Max}\left(\frac{1}{2}\left|V_{\alpha}+V_{\beta}\right|,-V_{\beta}\right)$ to $p_{i}$. In the equation, we have also included the Fermi factor

$$
F_{\beta}^{F}\left(p_{f}\right)=\left(1-\frac{1}{e^{\frac{E-\mu}{T}}+1}\right)
$$

which reflects the fact that inside the supernova some states have already been occupied by neutrinos.

Similarly, for $V_{\alpha}+V_{\beta}>0$, the process $\nu_{\alpha} \rightarrow \bar{\nu}_{\beta}+J$ can take place. The decay rate is given by Eq. (20) replacing $F_{\beta}^{F}\left(p_{f}\right)$ with $F_{\bar{\beta}}^{F}\left(p_{f}\right)$. The range of $p_{f}$ extends from $\operatorname{Max}\left(\frac{1}{2}\left|V_{\alpha}+V_{\beta}\right|, V_{\beta}\right)$ to $p_{i}$.

\subsection{2 $\nu+\nu \rightarrow J$ or $\bar{\nu}+\bar{\nu} \rightarrow J$}

In vacuum, the processes $\nu+\nu \rightarrow J$ or $\bar{\nu}+\bar{\nu} \rightarrow J$ are not kinematically allowed. However in medium, where $V$ is negative (positive) the process $\nu+\nu \rightarrow J(\bar{\nu}+\bar{\nu} \rightarrow J)$ can occur. Let us suppose $V<0$ and study the possibility of $\nu+\nu \rightarrow J$. Without loss of generality, we can write the four-momenta of the initial neutrinos as

$$
p_{1}=\left(p_{1}+V, 0,0, p_{1}\right) \quad p_{2}=\left(p_{2}+V, p_{2} \sin \theta, 0, p_{2} \cos \theta\right)
$$

for which $p_{1}+V$ and $p_{2}+V$ are both positive. Energy-momentum conservation implies

$$
p_{J}=\left(p_{1}+p_{2}+2 V, p_{2} \sin \theta, 0, p_{1}+p_{2} \cos \theta\right) .
$$

Recalling that we have neglected the effective mass of Majoron, the process $\nu\left(p_{1}\right)+\nu\left(p_{2}\right) \rightarrow$ $J\left(p_{J}\right)$ will be kinematically allowed if and only if $p_{J}^{2}=0$ or

$$
1-\cos \theta=-\frac{2 V^{2}+2 V\left(p_{1}+p_{2}\right)}{p_{1} p_{2}}
$$

which can be satisfied for $p_{1}, p_{2}>|V|$.

Neglecting $V^{2} / p^{2}$ effects, for $V_{\alpha}+V_{\beta}<0$, it can be shown that the cross section of $\nu_{\alpha} \nu_{\beta} \rightarrow J$ is given by

$$
\sigma=\frac{(2 \pi)\left|g_{\alpha \beta}\right|^{2}}{4 p_{1}^{2} p_{2}^{2}\left|v_{1}-v_{2}\right|}\left(p_{1}+p_{2}\right)\left|V_{\alpha}+V_{\beta}\right| \delta\left(\cos \theta-\cos \theta_{0}\right)
$$

where $p_{1}$ and $p_{2}$ are the momenta of the two initial particles, $\theta$ is the angle between them and $\cos \theta_{0}=1-\frac{\left|V_{\alpha}+V_{\beta}\right|\left(p_{1}+p_{2}\right)}{p_{1} p_{2}}$.

Similarly, it can be shown that for $V_{\alpha}+V_{\beta}>0$, instead of $\nu_{\alpha}+\nu_{\beta} \rightarrow J$, the process $\bar{\nu}_{\alpha}+\bar{\nu}_{\beta} \rightarrow J$ can take place with the cross section again given by Eq. (22). 


\subsubsection{The process $\nu+\bar{\nu} \rightarrow J+J$}

For reasons that will become clear in a moment, we analyze $\nu$ and $\bar{\nu}$ as wave packets rather than as plane waves. Let us ignore the neutrino mass for simplicity. Then, calculating diagram (c) in figure 2, we find

$$
\begin{gathered}
2 \pi i \mathcal{M}=(2 \pi)^{4}(i g)(2 \pi)^{4}\left(i g^{*}\right) \frac{1}{(2 \pi)^{6}} \frac{1}{\sqrt{4 k_{1}^{0} k_{2}^{0}}} \times \iint f\left(p_{2}\right) u^{T}\left(p_{2}\right) \sigma_{2} \frac{1}{(2 \pi)^{4}} \times \\
\left(\frac{q^{0}-V-\vec{q} \cdot \vec{\sigma}}{\left(q^{0}-V\right)^{2}-|\vec{q}|^{2}+i \epsilon}-2 \pi\left\{\theta\left(-q^{0}\right)+\epsilon\left(q^{0}\right)\left(1-F^{F}\left(q^{0}\right)\right)\right\} \delta\left[\left(q^{0}\right)^{2}-(q+V)^{2}\right]\right) \\
\times \sigma_{2} \nu\left(p_{1}\right) \bar{f}\left(p_{1}\right) d^{3} p_{1} d^{3} p_{2}+\left(k_{1} \leftrightarrow k_{2}\right),
\end{gathered}
$$

where $k_{1}$ and $k_{2}$ are the momenta of the Majorons and $\int f\left(p_{2}\right)\left|p_{2}\right\rangle d^{3} p_{2}$ and $\int \bar{f}\left(p_{1}\right)\left|p_{1}\right\rangle d^{3} p_{1}$ represent the states of the neutrino and anti-neutrino, respectively. In Eq. (23), $q=k_{2}-p_{2}$ and we have considered the matter effects in the propagator:

$$
F^{F}\left(q^{0}\right)=1-1 /\left[\exp \left(\left(q^{0}-\mu\right) / T\right)+1\right]
$$

is the Fermi factor.

For both positive and negative $V$, in the vicinity of $\left(\overrightarrow{k_{1}}=\overrightarrow{p_{1}}, \overrightarrow{k_{2}}=\overrightarrow{p_{2}}\right)$ and $\left(\overrightarrow{k_{1}}=\overrightarrow{p_{2}}, \overrightarrow{k_{2}}=\right.$ $\overrightarrow{p_{1}}$ ), there are poles which are non-integrable singularities. Without the wave packets, the total cross section would be divergent. Setting $m_{\nu}$ non-zero just shifts the pole a little bit and does not solve this problem. This is due to the fact that for negative (positive) $V$, the processes $\bar{\nu} \rightarrow \nu+J$ and $\nu+\nu \rightarrow J(\nu \rightarrow \bar{\nu}+J$ and $\bar{\nu}+\bar{\nu} \rightarrow J)$ can take place on shell, so the singularity is indeed a physical one. Essentially, for $V<0$ the reaction $\nu \bar{\nu} \rightarrow J J$ can proceed in two steps, first, $\bar{\nu} \rightarrow \nu J$ and later, at a completely distant place $\nu \nu \rightarrow J$. In other words, the total cross section has two parts: i) a "connected" part; ii) a "disconnected" part which can be considered as two successive three-point processes.

Let us now consider in more detail the relation between Eq. (23) and its component three-point processes. For definiteness, we consider the case $V<0$. We have explained that the reaction $\bar{\nu} \nu \rightarrow J J$ contains a subprocess that factorizes as

$$
\int_{q}\left\langle J_{1} J_{2} \mid \nu \nu(q) J_{1}\right\rangle\left\langle\nu(q) J_{1} \mid \bar{\nu}\right\rangle .
$$

More explicitly, the factorized amplitude takes the form

$$
2 \pi i \mathcal{M}=\frac{-g g^{*}}{(2 \pi)^{6} \sqrt{4 k_{1}^{0} k_{2}^{0}}} \iiint \iint_{\tau}^{2 \tau} \int_{-\tau}^{\tau} f\left(p_{2}\right) u\left(p_{2}\right) e^{\left(\left(\vec{p}_{2}-\vec{k}_{2}\right) \cdot \vec{x}_{2}-\left(p_{2}^{0}-k_{2}^{0}\right) x_{2}^{0}\right)}
$$




$$
\begin{gathered}
\left.\sigma_{2} \frac{1}{(2 \pi)^{3}} \int \frac{q^{0}-V-\vec{\sigma} \cdot \vec{q}}{2|\vec{q}|} F^{F}\left(q^{0}\right) e^{-i q^{0}\left(x_{2}^{0}-x_{1}^{0}\right)} e^{i \vec{q} \cdot\left(\vec{x}_{2}-\vec{x}_{1}\right)} \delta\left(q^{0}-V-|\vec{q}|\right) d^{4} q\right) \\
\sigma_{2} v\left(p_{1}\right) \bar{f}\left(p_{1}\right) e^{i\left(\left(\vec{p}_{1}-\vec{k}_{1}\right) \cdot \vec{x}_{1}-\left(p_{1}^{0}-k_{1}^{0}\right) x_{1}^{0}\right)} d^{3} p_{1} d^{3} p_{2} d x_{1}^{0} d x_{2}^{0} d^{3} x_{1} d^{3} x_{2},
\end{gathered}
$$

where $F^{F}\left(q^{0}\right)=1-1 /\left(\exp \left(\left(q^{0}-\mu\right) / T\right)+1\right)$ represents the matter effects. In Eq. (24), $\tau$ represents the boundaries on time integrations and therefore it must be very large (i.e., $\tau \gtrsim 5 / V)$. We have written the time boundaries explicitly to emphasize the causality conditions. Transferring the amplitude for $\bar{\nu} \nu \rightarrow J J$ (Eq. (23)) from momenta $p_{1}, p_{2}$ to coordinates $x_{1}, x_{2}$, it can be shown that for the region $\left|x_{2}-x_{1}\right|>2 \tau \gtrsim 10 / V$, these two correspond. Therefore, if the initial neutrino and anti-neutrino are localized at distance $R>10 / V$, their interaction rate can be calculated by Eq. (24) instead of Eq. (23).

Consider $\nu$ and $\bar{\nu}$ which are localized at distance $R>10 / V$ far from each other. We have shown that their interaction cross section is given by $\left|\left\langle J_{1} J_{2} \mid \nu \nu J_{1}\right\rangle\left\langle\nu J_{1} \mid \bar{\nu}\right\rangle\right|^{2}$. So, this interaction can be considered as two subsequent processes. First $\bar{\nu}$ decays into $J_{1}$ and $\nu$. Then, the produced neutrino propagates a distance $R$ and annihilates with the other $\nu$ into $J_{2}$. In other words, to calculate the interaction probability of two such states, we can consider $\bar{\nu} \rightarrow \nu J$ as an additional source for $\nu$ and consequently the process $\nu \nu \rightarrow J$. This can be compared to the more familiar sources of neutrinos like electron capture, $\langle J \mid \nu \nu\rangle\left\langle\nu n \mid e^{-} p^{+}\right\rangle$.

Of course the set of states that are localized at distance $R>10 / V \sim 10^{-9} R_{\text {core }}$ far from one another is not a complete set. We should also consider the states which are closer and/or have overlap with each other. If we rewrite Eq. (23) in the x-coordinates, as we have done in Eq. (24), calculation of the amplitude of two states localized next to each other at distance $R$ will be easier. For such two states, the integral for $\left|\overrightarrow{x_{1}}-\overrightarrow{x_{2}}\right|>10 / V$ vanishes (because of the specific form of $f\left(p_{1}\right)$ and $\bar{f}\left(p_{2}\right)$ ), so we can restrict the integration over $\left|\overrightarrow{x_{1}}-\overrightarrow{x_{2}}\right|$ to the interval $(0,10 / V)$. For $\left|q^{0}-V-\right| \vec{q}|| \gg|V| / 10$, the amplitude for two states localized at $R<10 / V$ far from each other is equal to the amplitude for states with definite momenta, but for $\left|q^{0}-V-\right| \vec{q}||<|V| / 10$, the amplitude for the two localized states is much smaller. This is because in calculation of amplitude for two states with definite momenta, we encounter an integration $\int_{0}^{\infty} g(x) e^{i\left(q^{0}-V-|\vec{q}|\right) x} d x$ which diverges for $q^{0}-V-|\vec{q}| \rightarrow 0$ but for two states which are localized next to each other the corresponding integration is $\int_{0}^{10 / V} g(x) e^{i\left(q^{0}-V-|\vec{q}|\right) x} d x$ which is finite. The total cross section for neutrinos and antineutrinos localized next to each other is then given by an angular integral over the square of (23) in which the integration is over all angles except those for which $\left|q^{0}-V-\right| \vec{q}||<|V| / 10$. Consider the special case that the sum of the momenta of $\nu$ and $\bar{\nu}$ is zero. Setting the cutoff 
equal to $\lambda|V| / 10,(\lambda$ is an arbitrary number of order of one) for such two particles we obtain

$$
\sigma_{t o t} \sim \frac{|g|^{4}}{8 \pi p_{1} p_{2}\left|v_{1}-v_{2}\right|}\left[\ln \left(\frac{p_{1} p_{2}}{(\lambda V / 10)^{2}}\right)+\frac{10}{\lambda}-\frac{14}{4}\right] .
$$

Since we have a preferred frame (the frame of the supernova), the total cross section is not Lorentz invariant and for each $\nu$ and $\bar{\nu}$ the total cross section must be calculated, independently. Now consider a pair of a neutrino and an anti-neutrino that make a general angle. Then Eqs. $(19,21)$ show that in the vicinity of singularity the momentum flowing in the propagator is of the order of $|V|$ (i.e., $|\vec{q}|=\left|\vec{p}_{2}-\vec{k}_{2}\right| \sim|V|$ ). Therefore for scattering angles that $\left(q^{0}-V-\vec{q} \cdot \vec{\sigma}\right) /\left[\left(q^{0}-V\right)^{2}-|\vec{q}|^{2}\right] \sim 1 / V$, the phase factor $\left(\int d^{3} k_{1} d^{3} k_{2} \delta^{4}\left(p_{1}+p_{2}-\right.\right.$ $\left.k_{2}-k_{1}\right)$ ) is of the order of $|V|^{2} / p^{2}$. Thus the total cross section has no strong dependence on $|V|$ and for general initial momenta the cross section can be estimated by Eq. (25).

Here, for simplicity we have dropped the flavor indices but for the more general case the discussion is similar.

\subsubsection{The process $\nu+\nu \rightarrow J+J$ and $\bar{\nu}+\bar{\nu} \rightarrow J+J$}

The discussion of $\nu \nu \rightarrow J J$ and $\bar{\nu}+\bar{\nu} \rightarrow J+J$ can be carried out in a similar way. For quasi-degenerate neutrino masses, the amplitude for $\nu \nu \rightarrow J J$ (see diagram (d) in Fig. (2)) is given by,

$$
\begin{gathered}
\frac{1}{(2 \pi)^{2} \sqrt{4 k_{1}^{0} k_{2}^{0}}} \sum_{\gamma} \iint f_{1}\left(p_{1}\right) u_{\alpha}^{T}\left(p_{2}\right) C\left(i g_{\alpha \gamma}\right)\left(i g_{\gamma \beta}\right) \times \\
\frac{i m\left(m^{2}+V_{\gamma}^{2}+q^{2}-q_{0}^{2}+2 \vec{q} \cdot \vec{\sigma} V_{\gamma}\right)}{\left(m^{2}-q_{0}^{2}+\left(V_{\gamma}-q\right)^{2}\right)\left(m^{2}-q_{0}^{2}+\left(V_{\gamma}+q\right)^{2}\right)} \times \\
f_{2}\left(p_{2}\right) u\left(p_{2}\right) d^{3} p_{1} d^{3} p_{2}+\left(k_{1} \leftrightarrow k_{2}\right)+\mathcal{A},
\end{gathered}
$$

where $\int f_{1}\left(p_{1}\right)\left|p_{1}\right\rangle d^{3} p_{1}$ and $\int f_{2}\left(p_{2}\right)\left|p_{2}\right\rangle d^{3} p_{2}$ represent the initial neutrino states, $k_{1}$ and $k_{2}$ are the momenta of the emitted Majorons and $q=k_{2}-p_{2}$. The term $\mathcal{A}$ summarizes all of the Fermi effects on the propagator. The amplitude for values of $q$ which $\left(q+V_{\gamma}\right)^{2}-q_{0}^{2}-m^{2} \sim$ $p_{1}^{2}, p_{2}^{2} \gg m^{2}, V_{\gamma}^{2}$ is negligible, and the main contribution to the cross section comes from the small solid angle $\left(\sim V^{2} / p_{1} p_{2}\right)$ for which $(q+V)^{2}-q_{0}^{2}-m^{2} \lesssim V^{2}$.

First, let us discuss the process $\nu_{e} \nu_{e} \rightarrow J J$. In general, for $\gamma=\mu, \tau$, there are singularities which correspond to an on-shell $\nu_{\mu}$ or $\nu_{\tau}$. Note that, if $\overrightarrow{p_{1}}$ and $\overrightarrow{p_{2}}$ are parallel or make an angle smaller than $\sim\left|V_{e} / V_{\mu}\right|$, the singularities disappear. As for the case $\bar{\nu} \nu \rightarrow J J$, we can discuss that if the initial states are localized at distance $R>10 / V_{\mu}$ far from each other, the process $\nu \nu \rightarrow J J$ will be equivalent to two successive processes $\left\langle\nu_{\mu(\tau)} J \mid \nu_{e}\right\rangle$ and then 
$\left\langle J \mid \nu_{e} \nu_{\mu(\tau)}\right\rangle$. This yields a cutoff of $V_{\mu} / 10$ for calculating the 4-point total cross-section. Note that although $\nu_{e} \rightarrow J+\nu_{\mu}$ is kinematically allowed $\left(V_{\mu}<V_{e}\right), \Gamma\left(\nu_{e} \rightarrow J \nu_{\mu}\right)$ is suppressed by $\left(m / p_{\nu_{e}}\right)^{2}$ and in practice, will not have any significant effect.

For $\gamma=e$, there is no singularity (except for the case that one of the Majorons is soft and $\nu_{e} \nu_{e} \rightarrow J$ is kinematically possible) and therefore no cutoff is needed. The total cross section can be estimated as

$$
\sigma_{t o t}\left(\nu_{e} \nu_{e} \rightarrow J J\right)=\frac{1}{\left|v_{1}-v_{2}\right|(2 \pi)^{2} p_{1} p_{2}}\left(a\left|g_{e \mu}^{2}+g_{e \tau}^{2}\right|^{2}\left(\frac{m}{V_{\mu}}\right)\left(\frac{m}{V_{\mu} / 10}\right)+b\left|g_{e e}\right|^{4}\right) .
$$

Similarly,

$$
\begin{gathered}
\left.\sigma_{t o t}\left(\nu_{e} \nu_{\mu(\tau)}\right) \rightarrow J J\right)=\frac{1}{\left|v_{1}-v_{2}\right|(2 \pi)^{2} p_{1} p_{2}} \times \\
\left(a^{\prime}\left|g_{e \mu} g_{\mu \mu(\tau)}+g_{e \tau} g_{\tau \mu(\tau)}\right|^{2}\left(\frac{m}{V_{\mu}}\right)\left(\frac{m}{V_{\mu} / 10}\right)+b^{\prime}\left|g_{e e} g_{e \mu(\tau)}\right|^{2}\right)
\end{gathered}
$$

and

$$
\sigma_{t o t}\left(\nu_{\mu(\tau)} \nu_{\mu(\tau)} \rightarrow J J\right)=\frac{1}{\left|v_{1}-v_{2}\right|(2 \pi)^{2} p_{1} p_{2}}\left(a^{\prime \prime}\left|g_{\mu \mu(\tau)}^{2}+g_{\tau \mu(\tau)}^{2}\right|^{2}+b^{\prime \prime}\left|g_{e \mu(\tau)}\right|^{4}\right) .
$$

with $b, b^{\prime}, b^{\prime \prime}, a, a^{\prime}$ and $a^{\prime \prime}$ of order 1. In Ref. [20], $\sigma_{t o t}\left(\nu_{e} \nu_{e} \rightarrow J J\right)$ has been calculated, ignoring $V$ and the off-diagonal elements of the coupling matrix. The result agrees with our estimation in the sense that the term proportional to $\left|g_{e e}\right|^{4}$ is not suppressed by $m$.

The total cross section for $\left(\bar{\nu}_{\alpha} \bar{\nu}_{\beta} \rightarrow J J\right)$ is equal to $\sigma_{t o t}\left(\nu_{\alpha} \nu_{\beta} \rightarrow J J\right)$ replacing $V$ with $(-V)$.

\subsubsection{The processes $\nu+J \rightarrow \bar{\nu}$ or $\bar{\nu}+J \rightarrow \nu$ :}

These processes are the opposite of anti-neutrino and neutrino decay and, hence, the kinematical conditions are similar. If $V_{\alpha}+V_{\beta}$ is negative (positive) the process $\nu_{\alpha} J \rightarrow \bar{\nu}_{\beta}($ $\left.\bar{\nu}_{\alpha} J \rightarrow \nu_{\beta}\right)$ can take place with cross section

$$
\sigma=\frac{(2 \pi)}{4 p q\left|v_{1}-v_{2}\right|}\left|g_{\alpha \beta}\right|^{2} \frac{\left|V_{\alpha}+V_{\beta}\right|}{p} F_{(-)}^{F}(p+q) \delta\left(\cos \theta-\cos \theta_{0}\right)
$$

where $p$ and $q$ are the momenta of the initial neutrino and Majoron, respectively. $\theta$ is the angle between the two initial states and

$$
\cos \theta_{0}=-1+\frac{(p+q)\left|V_{\alpha}+V_{\beta}\right|}{p q} .
$$

$\underset{\beta}{F_{(-)}^{F}}(p+q)$ is the Fermi factor for the final state. 


\subsubsection{The Majoron decay, $J \rightarrow \nu+\nu$ or $J \rightarrow \bar{\nu}+\bar{\nu}$}

The decay $J \rightarrow \nu \nu(J \rightarrow \bar{\nu} \bar{\nu})$ is the opposite of the interaction $\nu \nu \rightarrow J(\bar{\nu} \bar{\nu} \rightarrow J)$ and therefore the kinematics are similar.

For $V_{\alpha}+V_{\beta}<0\left(V_{\alpha}+V_{\beta}>0\right)$ the Majoron can decay into $\nu_{\alpha}+\nu_{\beta}\left(\bar{\nu}_{\alpha}+\bar{\nu}_{\beta}\right)$ and the decay rate up to a $\left(|V| / p_{i}\right)^{2}$ correction is given by

$$
d \Gamma=\frac{\left|g_{\alpha \beta}\right|^{2}}{8 \pi} \frac{\left|V_{\alpha}+V_{\beta}\right|}{p_{i}} \int_{0}^{p_{i}} F_{\alpha}^{F}\left(p_{f}\right) F_{\beta}^{F}\left(p_{i}-p_{f}\right) d p_{f}
$$

where $p_{i}$ and $p_{f}$ are the momenta of the Majoron and either of the final neutrinos, respectively. $F_{\alpha}^{F}$ and $F_{\beta}^{F}$ are the Fermi factors reflecting the fact that in the core of the supernova some states have been occupied by already present neutrinos.

\subsubsection{The processes $\nu+J \rightarrow \bar{\nu}+J$ and $\bar{\nu}+J \rightarrow \nu+J$}

The amplitude for $\nu_{e}+J \rightarrow \bar{\nu}_{e}+J$ has two singularities in the $t$-channel due to $\nu_{\mu}$ exchange. Using a similar discussion to that in section 2.2.4, it can be shown that these singularities may be considered as two successive three-point interactions $\left\langle\bar{\nu}_{e} \mid J \nu_{\mu}\right\rangle\left\langle\nu_{\mu} J \mid \nu_{e}\right\rangle$ and $\left\langle J \mid \nu_{e} \nu_{\mu}\right\rangle\left\langle\bar{\nu}_{e} \nu_{\mu} \mid J\right\rangle$. This yields a cutoff $\sim\left|V_{\mu}\right| / 10$ around the singularity to determine the four-point interaction. In the case of head-on collision where the initial particles are within a small solid angle $\sim(V / p)^{2} \ll 4 \pi$ around $\cos \theta=-1$, there will be another singularity in the $s$-channel which can be considered as $\left\langle\bar{\nu}_{e} J \mid \bar{\nu}_{\mu}\right\rangle\left\langle\bar{\nu}_{\mu} \mid \nu_{e} J\right\rangle$. We recall that any discussion about $\nu_{\mu}$ applies to $\nu_{\tau}$ as well, because these states are completely equivalent for the supernova evolution. The total cross-section for $\nu_{e} J \rightarrow \bar{\nu}_{e} J$ can be evaluated as

$$
\frac{1}{(2 \pi)^{2}\left|v_{1}-v_{2}\right| p_{1} p_{2}}\left(a\left|g_{e \mu}^{2}+g_{e \tau}^{2}\right|^{2}\left(\frac{m^{2}}{V_{\mu}^{2} / 10}\right)+b\left|g_{e e}\right|^{4}\right) F^{F}
$$

where $a \sim b \sim 1$ and $F^{F}$ is the Fermi-blocking factor for the final neutrino. A similar discussion holds for $\bar{\nu}_{e} J \rightarrow \nu_{e} J$, and the corresponding cross-section is also of the form of Eq. (32).

The processes $\nu_{\mu}+J \rightarrow \bar{\nu}_{e}+J, \nu_{e}+J \rightarrow \bar{\nu}_{\mu}+J, \bar{\nu}_{\mu}+J \rightarrow \nu_{e}+J$ and $\bar{\nu}_{e}+J \rightarrow \nu_{\mu}+J$ also have singularities in the $t$-channel due to $\nu_{\mu}$-exchange and can be considered as two successive three-point processes. Following the same discussion as in sections 2.2.3 and 2.2.4, we use the cutoff $\sim V_{\mu} / 10$ to evaluate the cross section for the four-point interactions. The cross-sections of these processes have the form

$$
\frac{1}{(2 \pi)^{2}\left|v_{1}-v_{2}\right| p_{1} p_{2}}\left(a\left|g_{e \mu} g_{\mu \mu}+g_{e \tau} g_{\mu \mu}\right|^{2}\left(\frac{m^{2}}{V_{\mu}^{2} / 10}\right)+b\left|g_{e e} g_{e \mu}\right|^{2}\right) F^{F},
$$


where $a \sim b \sim 1$ and $F^{F}$ is the Fermi-blocking factor for the final neutrinos. The processes $\nu_{\mu} J \rightarrow \bar{\nu}_{e} J$ and $\bar{\nu}_{e} J \rightarrow \nu_{\mu} J$ can also have singularities in the $s$-channel only if the initial particles are almost parallel, i.e., if their relative angle resides within a small solid angle $\sim(V / p)^{2} \ll 4 \pi$ around $180^{\circ}$. We can safely neglect such states.

For the process $\nu_{\mu} J \rightarrow \nu_{\mu} J$, there is no singularity and it is straightforward to show that the cross section is of the form,

$$
\frac{1}{(2 \pi)^{2}\left|v_{1}-v_{2}\right| p_{1} p_{2}}\left(a\left|g_{\mu \mu}^{2}+g_{\mu \tau}^{2}\right|+b\left|g_{\mu e}\right|^{2}\right) .
$$

\subsubsection{The processes $\nu+J \rightarrow \nu+J$ and $\bar{\nu}+J \rightarrow \bar{\nu}+J$}

In general, the process $\nu+J \rightarrow \nu+J$ has a singularity in the $t$-channel. With a similar discussion as in section 2.2.3, we can show that this singularity can be evaluated as two successive three-point interactions $\langle J \mid \nu \nu\rangle\langle\nu \nu \mid J\rangle$ resulting in a cutoff of the order of $V / 10$ for evaluation of the four-point interactions. Using this cutoff, the cross section is of the order of

$$
\frac{|g|^{4}}{(2 \pi)^{5}\left|v_{1}-v_{2}\right| p_{1} p_{2}} \ln \left(\frac{p_{1} p_{2}}{V^{2} / 100}\right) F^{F}
$$

where $F^{F}$ is the Fermi-blocking factor for the final neutrino.

If the initial particles undergo a head-on collision (i.e., they are within a small solid angle $\sim(V / p)^{2}$ around $\left.180^{\circ}\right)$ there will be another singularity in the $s$-channel which can be considered as $\langle\nu J \mid \bar{\nu}\rangle\langle\bar{\nu} \mid \nu J\rangle$. The process $\bar{\nu}+J \rightarrow \bar{\nu}+J$ has one singularity which can be evaluated as $\langle\bar{\nu} \mid J \nu\rangle\langle J \nu \mid \bar{\nu}\rangle$. Again the cross section is of the form of Eq. (35).

\section{Supernova core without Majorons}

The dynamics of a supernova explosion is described in a number of articles and books (e.g., [22]). Here we only review the aspects of the supernova explosion which are relevant for our calculations.

Very massive stars $\left(\mathcal{M}>8 \mathcal{M}_{\odot}\right)$, at the end of their lifetime, develop a degenerate core with a mass around $1.5 \mathcal{M}_{\odot}$ made up of iron-group elements. As the outer layer burns, it deposits more iron that adds to the mass of the core. Eventually the core reaches its Chandrasekhar limit, at which the Fermi-pressure of the electron gas inside the core cannot support the gravitational pressure, and the star collapses. The collapse forces nuclei to absorb the electrons via $e^{-}+p \rightarrow n+\nu_{e}$. At the early stages, the produced $\nu_{e}$ can escape from the 
core but, eventually, the core becomes so dense that even neutrinos are trapped. The layer beyond which neutrinos can escape without scattering is called the "neutrino-sphere".

As the density of the central core reaches nuclear density $\left(\rho \simeq 3 \times 10^{14} \mathrm{~g} / \mathrm{cm}^{3}\right)$, a shock wave builds up which propagates outwards. We will refer to the pre-shock stage as the infall stage. This stage takes only around $0.1 \mathrm{sec}$. As the shock wave reaches the neutrino-sphere, it dissociates the heavy nuclei. The dissociation has three different results:

1. It consumes the energy of the shock, so that the shock eventually stalls;

2. It allows neutrinos to escape more easily;

3. It liberates protons that interact with the electrons present in the star $\left(e^{-}+p \rightarrow n+\nu_{e}\right)$, giving rise to the famous "prompt $\nu_{e}$ burst". The prompt $\nu_{e}$ burst deleptonizes the star but carries only a few percent of the total energy.

The stalled shock should regain its energy. Otherwise, it cannot propagate further and give rise to the spectacular fireworks. According to the models, this energy is provided by $\nu_{e}$ diffusing from the inner core to outside. The density of $\nu_{e}$ inside the inner core is very high. The corresponding Fermi energy is $\sim 200 \mathrm{MeV}$ while the temperature is only around $10 \mathrm{MeV}$. At the beginning the temperature of the neutrino-sphere is around $20 \mathrm{MeV}$. So the diffused neutrinos leave their energy as they travel outside, warming up the core. This energy can revive the shock. (In fact, this mechanism is controversial [22], but we will not use the shock revival mechanism for our calculations. Most of our calculations are related to the inner core, which is free of these controversies.) The temperature in the outer core increases to $40 \mathrm{MeV}$; actually, the outer core and the neutrino-sphere become warmer than the center. At the outer core, neutrinos of each type $\left(\nu_{e}, \bar{\nu}_{e}, \nu_{\mu}, \bar{\nu}_{\mu}, \nu_{\tau}\right.$ and $\left.\bar{\nu}_{\tau}\right)$ are present. These neutrinos escape the star and deplete its binding energy $\left(E_{b}=(1.5-4.5) \times 10^{53} \mathrm{erg}\right.$ [18]).

Two kinds of "upper" bounds can be imposed on the neutrino-Majoron couplings by studying supernova evolution:

1) If the coupling constant is too large, the process $\nu_{e} \rightarrow J+\bar{\nu}_{e}$, during the infall stage, deleptonizes the core and according to the models [27] a successful explosion cannot occur. This bound has been correctly studied in [14, 15] and the result is $g_{e e} \lesssim 2 \times 10^{-6}$.

2) If the coupling is non-zero, Majorons can be produced inside the inner core and can escape freely from the star, depleting the binding energy. The observed neutrino pulse from SN1987a coincides with that predicted by current supernova models. This means that the energy carried away by Majorons (or any other exotic particles) should be smaller than 


\begin{tabular}{|c|c|c|}
\hline $\mathrm{t}(\mathrm{sec})$ & $V_{e} \quad(\mathrm{eV})$ & $V_{\mu}=V_{\tau} \quad(\mathrm{eV})$ \\
\hline 0 & 2.3 & -11.7 \\
0.5 & 1 & -12.3 \\
1 & -0.3 & -12.8 \\
1.5 & -1 & -13.1 \\
\hline
\end{tabular}

Table 1: The values of the effective potentials at different instants after bounce without Majoron production.

the binding energy. The Majoron luminosity, $\mathcal{L}_{J}$, as large as $10^{53} \mathrm{erg} / \mathrm{sec}$ could significantly affect the neutrino pulse. Here, we will take $\mathcal{L}_{J}<3 \times 10^{53} \mathrm{erg} / \mathrm{sec}$ as a conservative maximum allowed value. This gives an upper bound on the coupling constants.

If the coupling of Majorons is larger than a "lower bound", the Majorons will be trapped so strongly that their luminosity will be small. We will discuss this case later.

Let us review the characteristics of the core. The inner core $\left(R<R_{\text {inner }} \sim 10 \mathrm{~km}\right)$ to a good approximation is homogeneous. The density in the inner core is around $5 \times 10^{14}$ $\mathrm{g} / \mathrm{cm}^{3}$. The distributions of all types of neutrinos follow the Fermi-Dirac formula with $T_{\text {inner }} \sim 10-30 \mathrm{MeV}$ and different chemical potentials [28, 29]. As mentioned earlier, the chemical potential for $\nu_{e}$ is around $200 \mathrm{MeV}$. So, inside the inner core, $\nu_{e}$ is degenerate while the density of $\bar{\nu}_{e}$ is negligible $\left(\mu_{\bar{\nu}_{e}}=-\mu_{\nu_{e}}=-200 \mathrm{MeV}\right)$. The suppression of the density of $\bar{\nu}_{e}$ is due to absorption on electrons. In the first approximation, the chemical potentials for $\stackrel{(-)}{\nu}$ and $\stackrel{(-)}{\nu_{\tau}}$ are equal to zero. In Ref. [30], it is shown that, because the interactions of $\nu_{\mu}$ and $\nu_{\tau}$ with matter are slightly stronger than the interactions of $\bar{\nu}_{\mu}$ and $\bar{\nu}_{\tau}$, their chemical potentials become nonzero: $\mu_{\nu_{\mu}} / T=\mu_{\nu_{\tau}} / T \simeq 5 T / m_{p}<1$. We will neglect $\mu_{\nu_{\mu}}$ and $\mu_{\nu_{\tau}}$ in our analysis. In fact the large uncertainty in the determination of the temperature affects our results more dramatically. The presence of $\mu$ in supernova can break the equivalence of $\nu_{\mu}$ and $\nu_{\tau}$. However, we neglect this effect and treat $\nu_{\mu}$ and $\nu_{\tau}$ in exactly the same way. In Table 1, we show the values of $V_{e}$ and $V_{\mu}\left(=V_{\tau}\right)$ at different instants after the bounce inside the inner core. The values of $Y_{e}$ and $Y_{\nu_{e}}$ are taken from Ref. [28].

Outside the inner core, $R_{\text {inner }} \sim 10 \mathrm{~km}<R<R_{\text {out }} \sim 15 \mathrm{~km}$, the density of $\nu_{e}$ 
is much lower, $\mu_{\nu_{e}} / T \lesssim 1$, but instead the density of $\bar{\nu}_{e}$ is higher than in the inner core. In fact, in the outer core $\left(R_{\text {inner }}<R<R_{\text {out }}\right)$, thermal equilibrium for neutrinos is only an approximation. To evaluate the role of the outer core in Majoron production, we set $\mu_{\nu_{e}}=\mu_{\nu_{\mu}}=\mu_{\nu_{\tau}}=0$. The density in the outer core drops from $5 \times 10^{14} \mathrm{~g} / \mathrm{cm}^{3}$ to $5 \times 10^{13}$ $\mathrm{g} / \mathrm{cm}^{3}$. The temperature in the outer core drops abruptly [28] such that $T\left(R=R_{\text {inner }}\right)=35$ $\mathrm{MeV}$ while $T\left(R=R_{\text {out }}\right) \sim 2 \mathrm{MeV}$.

Different models predict different values for parameters; e.g., the predictions of different classes of models for $T_{\text {inner }}$ vary from $10 \mathrm{MeV}$ to $30 \mathrm{MeV}$ [29]. Moreover the production of Majorons can distort the density distributions. Considering these uncertainties, the simplified model that we have invoked is justified. With this approach, we will be able to examine the prediction of all models for the Majoron luminosity.

\section{Bounds on coupling constants}

In this section we explore the role of Majorons in the cooling of the supernova core. In subsection 4.1, we derive an upper bound on $\left|g_{e e}\right|$ assuming the produced Majorons leave the core without being trapped. In subsection 4.2, we derive upper bounds on $g_{\mu \mu}, g_{\tau \tau}, g_{\mu e}$ and $g_{\tau e}$, again assuming Majorons leave the core immediately after production. In subsection 4.3, we show that for the couplings lower than the bounds we have derived, the four-point interactions are negligible. In subsection 4.4, we derive the limits above which Majorons become trapped.

\subsection{Bounds on $\left|g_{e e}\right|$}

As represented in Table 1, immediately after the bounce, $V_{e}$ is positive, but eventually $V_{e}$ decreases and becomes negative, while $V_{\mu}$ and $V_{\tau}$ are negative from the beginning. As long as $V_{e}>0$, the interactions $\nu_{e} \rightarrow \bar{\nu}_{e}+J$ and $\nu_{e} \rightarrow \nu_{\mu(\tau)}+J$ are kinematically allowed but the latter is suppressed by a factor of $(m / p)^{2} \lesssim 10^{-16}$. So we will consider only the interaction

$$
\nu_{e} \rightarrow \bar{\nu}_{e}+J
$$

This interaction depletes the energy of the core at a rate

$$
\mathcal{L}_{J}=\frac{\left|g_{e e}\right|^{2} V_{e} \mu_{\nu_{e}}^{4}}{12(2 \pi)^{3}} \times\left(4 / 3 \pi R_{\text {inner }}^{3}\right) .
$$


We should note that this interaction not only carries energy away but also deleptonizes the core.

$$
\frac{d Y_{L}}{d t}=-2 \Gamma Y_{\nu_{e}}=-2 \frac{g_{e e}^{2} V_{e}}{8 \pi} Y_{\nu_{e}}
$$

where we have used the fact that $n_{\bar{\nu}_{e}} \ll n_{\nu_{e}}$. We know that the core is in $\beta$-equilibrium. Since the rate of the $\beta$-interaction is faster than $\Gamma$ (rate of $\beta$-interactions $/ \Gamma \sim 48 \pi G_{F}^{2} \mu_{\nu_{e}}^{3} T^{2} / g_{e e}^{2} V_{e}$ ) and at equilibrium the density of electrons is one order of magnitude larger than that of neutrinos, we expect that the densities of the neutrinos are not affected by Majoron production. In other words, the Fermi energy, $\mu_{\nu_{e}}$, and $Y_{\nu_{e}}$ are still given by Ref. [28]. However, deleptonization by Majoron emission can affect $V_{e}$ dramatically because different terms in $V_{e} \propto\left(3 Y_{L}+Y_{\nu}-1\right) / 2$ cancel each other $\left(Y_{\nu} \ll Y_{L} \simeq 0.3\right)$. Therefore in the presence of Majoron emission, $V_{e}$ vanishes faster. Let us evaluate the maximum energy that can be carried away by Majorons through $\nu_{e} \rightarrow \bar{\nu}_{e}+J$ in the stage when $V_{e}$ is positive. To have an estimation, we can approximate

$$
\frac{d V_{e}}{d t}=-b V_{e}-a
$$

where

$$
b=\sqrt{2} \frac{3}{8 \pi} G_{F} \frac{\rho}{M_{N}}\left|g_{e e}\right|^{2} Y_{\nu}
$$

and $a$ reflects the deleptonization effect without Majoron emission. According to Table 1, $a \simeq 2.6 \mathrm{eV} / \mathrm{sec}$. If we neglect the variation of $Y_{\nu}, \rho$ and $a$ with time, we conclude that

$$
V_{e}(t)=\left(V_{e}(0)+\frac{a}{b}\right) e^{-b t}-\frac{a}{b}
$$

so that, after $t_{1}=(1 / b) \times \ln \left(V_{e}(0) b / a+1\right), V_{e}$ vanishes. The energy carried away by Majorons up to $t_{1}$ can be approximated as

$$
E_{V_{e}<0}=\frac{g_{e e}^{2} \mu_{\nu_{e}}^{4}}{12(2 \pi)^{3}} \times 4 / 3 \pi R_{\text {inner }}^{3} \times\left(\frac{V_{e}(0)}{b}-\frac{a}{b^{2}} \ln \frac{V_{e}(0) b+a}{a}\right) .
$$

For $g_{e e} \gtrsim 10^{-7}, E_{V_{e}<0}$ converges to $4 \times 10^{51}$ erg. Increasing $g_{e e}$ increases $\mathcal{L}_{J}$, but on the other hand, $V_{e}$ vanishes in a shorter period. It is easy to show that, for any value of $g_{e e}$,

$$
E_{V_{e}<0}<4 \times 10^{51} \text { erg } \ll E_{b} .
$$

Therefore the energy loss at this stage does not affect star's evolution and hence we do not obtain any bound.

As shown in Table 1, about one second after the core bounce $V_{e}$ turns negative. As we discussed earlier, in the presence of neutrino decay $V_{e}$ changes its sign even faster. In a medium with negative $V_{e}$, the decay $\nu_{e} \rightarrow \bar{\nu}_{e}+J$ is not kinematically allowed and instead 
$\bar{\nu}_{e} \rightarrow \nu_{e}+J$ can take place. However, we know that, in the inner core, the density of electron antineutrinos is quite low $\left(\mu_{\bar{\nu}_{e}} \sim-200 \mathrm{MeV}\right.$ while $\left.T \sim 10 \mathrm{MeV}\right)$ so this interaction will not have any role in the cooling of the inner core. In such a medium, energy will be carried away by process

$$
\nu_{e}+\nu_{e} \rightarrow J
$$

In previous literature the possibility of this interaction was not discussed. The interaction (40), diminishes the lepton number by two units. Again we see that $\mu_{\nu_{e}}$ and $Y_{\nu_{e}}$ will not be considerably affected by this process, but that $V_{e}$ will decrease faster. In contrast to the previous case, a faster decrease of $V_{e}$ is a positive feedback for the process and leads to the energy depletion. The energy carried away from the inner core via the process in Eq. (40) is now

$$
\mathcal{L}_{J}=\frac{7}{12}\left|g_{e e}\right|^{2}\left|V_{e}\right| \frac{\mu_{\nu_{e}}^{4}}{(2 \pi)^{3}} \times\left(\frac{4}{3} \pi R_{\text {inner }}^{3}\right) .
$$

To evaluate a conservative upper bound on $\left|g_{e e}\right|$, we set $\left|V_{e}\right|$ equal to $0.3 \mathrm{eV}, \mu_{\nu_{e}}=200$ $\mathrm{MeV}$ and $R=10 \mathrm{~km}$ then,

$$
\mathcal{L}_{J}=2\left|g_{e e}\right|^{2} \times 10^{66} \times\left(\frac{R_{\text {inner }}}{10 \mathrm{~km}}\right)^{3}\left(\frac{V_{e}}{0.3 \mathrm{eV}}\right)\left(\frac{\mu_{\nu_{e}}}{200 \mathrm{MeV}}\right)^{4} \quad \frac{\mathrm{erg}}{\mathrm{sec}}
$$

Around one second after the core bounce, the total neutrino luminosity, $\mathcal{L}_{\nu}$, is about $5 \times 10^{52}$ erg/sec. So, the condition $\mathcal{L}_{J}<3 \times 10^{53} \mathrm{erg} / \mathrm{sec}$ yields the conservative bound,

$$
\left|g_{e e}\right|<4 \times 10^{-7}\left(\frac{R_{\text {inner }}}{10 \mathrm{~km}}\right)^{-\frac{3}{2}}\left(\frac{V_{e}}{0.3 \mathrm{eV}}\right)^{-\frac{1}{2}}\left(\frac{\mu_{\nu_{e}}}{200 \mathrm{MeV}}\right)^{-2} .
$$

In Ref. [14], a bound on $\left|g_{e e}\right|$ is obtained by studying the energy loss via $\bar{\nu}_{e} \rightarrow \nu_{e}+J$ which mainly takes place in the outer core, $R_{\text {inner }} \simeq 10 \mathrm{~km}<R<R_{\text {out }} \simeq 20 \mathrm{~km}$. The result is $\mathcal{L}\left(\bar{\nu}_{e} \rightarrow \nu_{e}+J\right)=$ few $\times 10^{64}\left|g_{e e}\right|^{2} \mathrm{erg} / \mathrm{sec}$. So the conservative bound $\mathcal{L}\left(\bar{\nu}_{e} \rightarrow \nu_{e}+J\right)<3 \times 10^{53} \mathrm{erg} /$ sec implies $\left|g_{e e}\right|<4 \times 10^{-6}$. The bound in Eq. (42) is one order of magnitude stronger because the total number of $\nu_{e}$ in the inner core is very high. In Ref. [15], a bound is imposed due to the processes $\nu+\nu \rightarrow J+J$ and $\nu \rightarrow \nu+J(\nu$ denotes both neutrino and antineutrino). However the energy carried away is overestimated due to an improper treatment of the three-point subprocesses. We will elaborate on the $\nu+\nu \rightarrow J+J$ process in section 4.2 .

\subsection{Bounds on $\left|g_{\mu \alpha}\right|$ and $\left|g_{\tau \alpha}\right|$}

In this subsection we discuss the processes involving $\stackrel{(-)}{\nu_{\tau}}$ and/or $\stackrel{(-)}{\nu_{\mu}}$. These processes include

$$
\text { (a) } \quad \nu_{\mu, \tau}+\nu_{\mu, \tau} \rightarrow J, \quad \nu_{\mu, \tau}+\nu_{e} \rightarrow J
$$


and

$$
\text { (b) } \quad \bar{\nu}_{\mu, \tau} \rightarrow J+\nu_{e, \mu, \tau} .
$$

The process $\bar{\nu}_{\mu, \tau} \rightarrow J+\nu_{e}$ can take place only in the outer core where electron neutrinos are not degenerate. Both processes (a) and (b) can distort the distribution of matter inside the star. However, that calculation is beyond the scope of this paper. But we can argue that it is a good approximation, for the purpose of computing upper bounds, to use distributions with vanishing chemical potentials for $\nu_{\tau}$ and $\nu_{\mu}$ [28]. For simplicity we rotate $\left(\nu_{\mu}, \nu_{\tau}\right)$ to a basis such that $g_{\mu \tau}=0$. Note that since the chemical potential is diagonal and $V_{\mu}=V_{\tau}$, it will be invariant under this rotation. In the new basis, we can write, for the inner core

$$
\frac{d n_{\nu_{\mu}}}{d t}-\frac{d n_{\bar{\nu}_{\mu}}}{d t}=2\left[\operatorname{Rate}\left(\bar{\nu}_{\mu} \rightarrow \nu_{\mu}+J\right)-\operatorname{Rate}\left(\nu_{\mu} \nu_{\mu} \rightarrow J\right)\right]-\operatorname{Rate}\left(\nu_{\mu} \nu_{e} \rightarrow J\right),
$$

where we have neglected $\nu \nu \rightarrow J J$ interactions. The sum of the chemical potentials for $\nu_{\mu}$ and $\bar{\nu}_{\mu}$ must be zero, $\mu \equiv \mu_{\nu_{\mu}}=-\mu_{\bar{\nu}_{\mu}}$, therefore

$$
n_{\nu_{\mu}}=\int \frac{4 \pi}{(2 \pi)^{3}} \frac{p^{2} d p}{e^{\frac{p-\mu}{T}}+1} \text { while } n_{\bar{\nu}_{\mu}}=\int \frac{4 \pi}{(2 \pi)^{3}} \frac{p^{2} d p}{e^{\frac{p+\mu}{T}}+1} .
$$

We expect that for small values of $\left|g_{\alpha \beta}\right|$, the chemical potential remains small. Let us suppose $|\mu / T| \ll 1$ to solve the equation (43), then we can determine whether this assumption is valid or not. For $|\mu / T| \ll 1$,

$$
n_{\nu_{\mu}} \simeq \frac{4 \pi T^{3}}{(2 \pi)^{3}}(1.8+1.64 \mu / T), \quad n_{\bar{\nu}_{\mu}} \simeq\left(4 \pi T^{3} /(2 \pi)^{3}\right)(1.8-1.64 \mu / T)
$$

and we can rewrite the right hand side of Eq. (43) as

$$
\frac{\left|g_{\mu \mu}\right|^{2}\left|V_{\mu}\right| T^{3}}{2(2 \pi)^{3}}\left\{0.12-3.28 \mu / T-(0.34+0.25 \mu / T) \frac{\left|g_{e \mu}\right|^{2}}{\left|g_{\mu \mu}\right|^{2}} \frac{\left|V_{\mu}+V_{e}\right|}{\left|V_{\mu}\right|} \frac{\mu_{\nu_{e}}^{2}}{T^{2}}\right\},
$$

where $\mu_{\nu_{e}}$ is the chemical potential of the electron-neutrinos. Inside the supernova core, neutrinos and matter are in thermal equilibrium and since the energy density of matter is much higher, we expect that the rate of thermal change due to these processes is small:

$$
\left|\frac{d T}{T d t}\right| \sim\left|\frac{E_{\nu_{e}} d n_{\nu_{\mu}} / d t}{E_{b} / \text { volume }}\right| \ll \frac{d n_{\nu_{\mu}}}{n_{\nu_{\mu}} d t} .
$$

So, $d n_{\nu_{\mu}} / d t-d n_{\bar{\nu}_{\mu}} / d t \simeq 2 \frac{4 \pi T^{3}}{(2 \pi)^{3}} \times 1.64 d(\mu / T) / d t$. On the other hand, for this estimation we can neglect the variation in $V_{\mu} \simeq \sqrt{2} G_{F} n_{B}\left(Y_{e}-1\right) / 2$. Also, since the density of $\nu_{e}$ is much higher than that of $\nu_{\mu}$, we can neglect the variation of $\mu_{\nu_{e}}$. Therefore, Eq. (45) tells us that $\mu / T$ converges to

$$
\left(0.12-0.34 \frac{\left|g_{e \mu}\right|^{2}}{\left|g_{\mu \mu}\right|^{2}} \frac{\left|V_{e}+V_{\mu}\right|}{\left|V_{\mu}\right|} \frac{\mu_{\nu_{e}}^{2}}{T^{2}}\right) /\left(3.28+0.25 \frac{\left|g_{e \mu}\right|^{2}}{\left|g_{\mu \mu}\right|^{2}} \frac{\left|V_{e}+V_{\mu}\right|}{\left|V_{\mu}\right|} \frac{\mu_{\nu_{e}}^{2}}{T^{2}}\right) .
$$


Now, it is easy to show that for $\left(\left|g_{e \mu}\right|^{2} /\left|g_{\mu \mu}\right|^{2}\right) \times\left(\mu_{\nu_{e}}^{2} / T^{2}\right)<37,|\mu / T|$ remains small regardless of the values of $\left|g_{e \mu}\right|$ or $\left|g_{\mu \mu}\right|$, themselves. For $\left|g_{e \mu}\right|>6\left|g_{\mu \mu}\right| T / \mu_{\nu_{e}},|\mu / T|$ diverges to values larger than 1 and the above analysis is no longer correct (remember that we had assumed $|\mu / T| \ll 1)$. In this case, $\nu_{\mu}$ will disappear after $\sim\left(\frac{\left|g_{e \mu}\right|^{2}}{100 \pi} V_{\mu \mu} \mu_{\nu_{e}}^{2} / T^{2}\right)^{-1}$ but on the other hand, the density of $\bar{\nu}_{\mu}$ will increase (the chemical potential becomes negative) and this calls for recalculation of the density distributions. We can make a similar discussion for $\nu_{\tau}$. Let us suppose $\left|g_{e \mu}\right|<6\left|g_{\mu \mu}\right| T / \mu_{\nu_{e}}$ and $\left|g_{e \tau}\right|<6\left|g_{\tau \tau}\right| T / \mu_{\nu_{e}}$ and continue from here.

Now let us evaluate the Majoron luminosity using the distributions given in [28]. Neglecting the Majoron emission from the outer core we find,

$$
\mathcal{L}\left(\nu_{\alpha}+\nu_{\beta} \rightarrow J\right) \simeq \frac{2}{3} \frac{R_{\text {inner }}^{3}}{(2 \pi)^{2}} T_{\text {inner }}^{4}\left|g_{\alpha \beta}\right|^{2}\left(\left|V_{\alpha}+V_{\beta}\right|\right)
$$

and

$$
\mathcal{L}\left(\bar{\nu}_{\alpha} \rightarrow \nu_{\beta} J\right) \simeq \frac{1.3}{3} \frac{R_{\text {inner }}^{3}}{(2 \pi)^{2}} T_{\text {inner }}^{4}\left|g_{\alpha \beta}\right|^{2}\left(\left|V_{\alpha}+V_{\beta}\right|\right),
$$

where by $\alpha$ and $\beta$ we denote $\mu$ or $\tau$. On the other hand,

$$
\mathcal{L}\left(\nu_{\alpha}+\nu_{e} \rightarrow J\right) \simeq \frac{R_{\text {inner }}^{3}}{3(2 \pi)^{2}}\left|g_{\alpha e}\right|^{2}\left(\left|V_{\alpha}+V_{e}\right|\right)\left(0.2 \mu_{\nu_{e}}^{3} T_{\text {inner }}\right) .
$$

Note that even if $g_{\alpha e}$ is large, the process $\left(\bar{\nu}_{\alpha} \rightarrow \nu_{e}+J\right)$, in the inner core, is suppressed by a factor of $\exp \left(\left(T_{\text {inner }}-\mu_{\nu_{e}}\right) / T_{\text {inner }}\right)$ because inside the star, $\nu_{e}$ is degenerate.

Then, the requirement $\mathcal{L}<3 \times 10^{53} \mathrm{erg} / \mathrm{sec}$ implies

$$
\sqrt{\sum_{\alpha, \beta \in \mu, \tau}\left|g_{\alpha \beta}\right|^{2}} \lesssim 8 \times 10^{-7}\left(\frac{10 \mathrm{eV}}{\left|V_{\mu}\right|}\right)^{\frac{1}{2}}\left(\frac{20 \mathrm{MeV}}{T_{\text {inner }}}\right)^{2}\left(\frac{10 \mathrm{~km}}{R_{\text {inner }}}\right)^{\frac{3}{2}}
$$

and

$$
\sqrt{\left|g_{\mu e}\right|^{2}+\left|g_{\tau e}\right|^{2}}<5 \times 10^{-7}\left(\frac{10 \mathrm{~km}}{R_{\text {inner }}}\right)^{\frac{3}{2}}\left(\frac{200 \mathrm{MeV}}{\mu_{\nu_{e}}}\right)^{\frac{3}{2}}\left(\frac{20 \mathrm{MeV}}{T_{\text {inner }}}\right)^{\frac{1}{2}}\left(\frac{10 \mathrm{eV}}{V_{\mu}}\right)^{\frac{1}{2}} .
$$

We emphasize again that the above results are valid only assuming that $\left|g_{e \mu} \mu_{\nu_{e}} / g_{\mu \mu} T\right|^{2}<37$ and $\left|g_{e \tau} \mu_{\nu_{e}} / g_{\tau \tau} T\right|^{2}<37$. Otherwise the $\nu_{\mu(\tau)}$ annihilation will stall because $\nu_{\mu(\tau)}$ is depleted. Meanwhile, the energy carried away due to $\nu_{\mu(\tau)}$-annihilation is of the order of

$$
\mathcal{L}\left(\nu_{e}+\nu_{\mu(\tau)} \rightarrow J\right)\left(\frac{\left|g_{e \alpha}\right|^{2}}{100 \pi} V_{\mu} \frac{\mu_{\nu_{e}}^{2}}{T^{2}}\right)^{-1} \sim 10^{49} \text { erg } \ll E_{b} \sim 10^{53} \text { erg }
$$

So, $\mathcal{L}\left(\nu_{e}+\nu_{\mu(\tau)} \rightarrow J\right)$ does not impose any bound on $\left|g_{e \mu(\tau)}\right|$. On the other hand, since the density of $\bar{\nu}_{\mu(\tau)}$ grows, the process $\mathcal{L}\left(\bar{\nu}_{\mu(\tau)} \rightarrow \nu_{\mu(\tau)} J\right)$ will even become intensified and we expect that still Eq. (49) will be a conservative bound. For $\left(\left|g_{e \mu}\right|^{2} /\left|g_{\mu \mu}\right|^{2}\right) \times\left(\mu_{\nu_{e}}^{2} / T^{2}\right)>37$, 
the upper bound on $\left|g_{e \mu}\right|$ is imposed by $\bar{\nu}_{\mu}$-decay in the outer core. Using the distributions in Ref. [28], we can show that

$$
\mathcal{L}\left(\bar{\nu}_{\mu(\tau)} \rightarrow J+\nu_{e}\right)=\text { few } \times\left|g_{e \mu(\tau)}\right|^{2} \times 10^{64} \mathrm{erg} / \mathrm{sec}
$$

which implies

$$
\left|g_{e \mu}\right|,\left|g_{e \tau}\right|<\text { few } \times 10^{-6}
$$

In Figs. (1) and (2), all these bounds are schematically depicted for $T_{\text {inner }}=10 \mathrm{MeV}$ and $T_{\text {inner }}=20 \mathrm{MeV}$, respectively. The shadowed area represents the range of parameters for which $\mathcal{L}_{J}<3 \times 10^{53} \mathrm{erg} / \mathrm{sec}$. As shown in Fig. (4), for $T=20 \mathrm{MeV}$, the process $\nu_{e}+\nu_{\mu} \rightarrow J$

does not impose any bound on $\left|g_{e \mu}\right|$ because, for any value of $\left|g_{e \mu}\right|$ smaller than $\sqrt{37}\left|g_{\mu \mu}\right| T / \mu_{\nu_{e}}$ (where $\left|g_{\mu \mu}\right|$ is below its upper bound) it cannot give rise to a Majoron luminosity larger than the allowed value.

\subsection{Four-point interactions}

In this subsection we discuss the processes $\nu+\nu \rightarrow J+J$ and $\bar{\nu}+\bar{\nu} \rightarrow J+J$. As discussed in sections 2.2.3 and 2.2.4, we consider only the intrinsically connected contributions, the effects of three-particle sub-processes subtracted. Using the distributions in Ref. [28] and the formulae we have found in subsection 2.2.3, we obtain

$$
\mathcal{L}\left(\nu_{e}+\bar{\nu}_{\mu(\tau)} \rightarrow J+J\right) \sim \frac{\mu_{\nu_{e}}^{3} T_{\text {inner }}^{2}}{(2 \pi)^{4}}\left(\frac{4 \pi}{3} R_{\text {inner }}^{3}\right)\left|\sum_{\alpha} g_{e \alpha} g_{\mu(\tau) \alpha}^{*}\right|^{2}
$$

and

$$
\mathcal{L}\left(\nu_{\mu(\tau)}+\bar{\nu}_{\mu(\tau)} \rightarrow J+J\right) \sim \frac{T_{\text {inner }}^{5}}{(2 \pi)^{4}}\left(\frac{4 \pi}{3} R_{\text {inner }}^{3}\right)\left|\sum_{\alpha} g_{\mu(\tau) \alpha} g_{\mu(\tau) \alpha}^{*}\right|^{2}
$$

In the above equations, $\alpha$ runs over $\{e, \mu, \tau\}$. Using the distributions in Ref. [28] and the formulae we found in section 2.2.4, we obtain

$$
\begin{gathered}
\mathcal{L}\left(\nu_{e}+\nu_{e} \rightarrow J+J\right)=\frac{1}{(2 \pi)^{6}}\left(a \frac{m^{2}}{V_{\mu}^{2} / 10}\left|g_{e \mu}^{2}+g_{e \tau}^{2}\right|^{2}+b\left|g_{e e}\right|^{4}\right)\left(\frac{4 \pi}{3} R_{\text {inner }}^{3}\right) \mu_{\nu_{e}}^{5} \\
\mathcal{L}\left(\nu_{e}+\nu_{\mu(\tau)} \rightarrow J+J\right)= \\
\left(\frac{4 \pi}{3} R^{3}\right) \frac{\mu_{\nu_{e}}^{3} T_{\text {inner }}^{2}}{(2 \pi)^{6}}\left(a^{\prime}\left|g_{e \mu} g_{\mu \mu(\tau)}+g_{e \tau} g_{\tau \mu(\tau)}\right|^{2} \frac{m^{2}}{V_{\mu}^{2} / 10}+b^{\prime}\left|g_{e e} g_{e \mu(\tau)}\right|^{2}\right)
\end{gathered}
$$

and

$$
\mathcal{L}\left(\nu_{\alpha}+\nu_{\beta} \rightarrow J+J\right) \sim \mathcal{L}\left(\bar{\nu}_{\alpha}+\bar{\nu}_{\beta} \rightarrow J+J\right)=
$$




$$
\left(\frac{4 \pi}{3} R_{\text {inner }}^{3}\right) \frac{T_{\text {inner }}^{5}}{(2 \pi)^{6}}\left(a^{\prime \prime}\left|g_{\mu \mu(\tau)}^{2}+g_{\mu \mu(\tau)}^{2}\right|^{2}+b^{\prime \prime}\left|g_{e \mu(\tau)}\right|^{2}\right)
$$

where $m$ is the neutrino mass for quasi-degenerate mass schemes. If $\left|g_{\alpha \beta}\right|$ is smaller than the "upper" bounds in Eqs. $(42,49,52)$ the above luminosities are negligible. These luminosities become non-negligible only if the couplings are larger than $10^{-5}$ so they do not change the "upper" bounds. Eqs. (53-56) depend on combinations of couplings so in general it is rather difficult to compare them with the Majoron luminosity due to three-point processes (i. e., $\left.\mathcal{L}\left(\bar{\nu}_{\alpha} \rightarrow J \nu_{\beta}\right), \mathcal{L}\left(\nu_{\alpha} \nu_{\beta} \rightarrow J\right)\right)$. Let us suppose that all elements are zero except for a particular $g_{\alpha \beta}$. Then, Eqs. $(46,47,54,56)$ imply that for $\left|g_{\mu \mu}\right|<5 \times 10^{-3}$ the three-point processes are dominant. If all couplings, but $\left|g_{e e}\right|$ are zero Eqs. (41) and (55) show that as long as $\left|g_{e e}\right|<10^{-3}, \nu_{e} \nu_{e} \rightarrow J$ is dominant. Comparing Eqs. (53) and (55) with Eq. (51) reveals that as long as $\left|g_{e \mu}\right|<10^{-4}$ the process $\bar{\nu}_{\mu} \rightarrow \nu_{e} J$ dominates. We note that, for coupling constants of the order of the "lower" bound (for which the produced Majorons are trapped), the four-point processes can play a significant role.

\subsection{Majoron decay and scattering}

So far we have assumed that Majorons leave the star without undergoing any interaction or decay. Now we discuss the validity of this assumption. First, let us discuss the possibility of decay. (Note that although Majorons are massless particles, in a medium such as a supernova, in principle, they can decay.) For $\alpha, \beta \in\{\mu, \tau\}$,

$$
\Gamma\left(J(q) \rightarrow \nu_{\alpha}+\nu_{\beta}\right)=\frac{\left|g_{\alpha \beta}\right|^{2}\left(\left|V_{\alpha}+V_{\beta}\right|\right)}{8 \pi}(0.8-0.27),
$$

where 0.8 and 0.27 correspond to $q / T=10$ and $q / T=0.1$, respectively. So, the Majorons decay before leaving the core $(\Gamma>1 / R)$, only if

$$
\left|g_{\alpha \beta}\right| \gtrsim 10^{-5}
$$

Because of degeneracy of the inner core, only the energetic Majorons $\left(\left|E_{J}-\mu_{\nu_{e}}\right| / T \gtrsim 1\right)$ can decay into electron neutrinos (see Eq. (31)). It can be shown that

$$
\Gamma\left[J\left(q>2 \mu_{\nu_{e}}\right) \rightarrow \nu_{e}+\nu_{e}\right] \sim \frac{T\left|g_{e e}\right|^{2}\left|V_{e}\right|}{4 \pi \mu_{\nu_{e}}}
$$

and

$$
\Gamma\left[J\left(q>\mu_{\nu_{e}}\right) \rightarrow \nu_{e}+\nu_{\alpha}\right] \sim \frac{\left|g_{e e}\right|^{2}\left|V_{e}+V_{\mu}\right|}{8 \pi} .
$$


If

$$
\left|g_{e \mu}\right|>7 \times 10^{-6} \quad \text { and/or } \quad\left|g_{e e}\right|>5 \times 10^{-5} .
$$

Majorons that are produced in the center will decay before leaving the core. Note that, even beyond the neutrinosphere as long as $|V|$ is large enough, Majoron decay can take place. Now let us examine the interaction effect. For low values of coupling constants, the dominant interactions are $(\nu+J \rightarrow \bar{\nu})$ with the mean free path

$$
\begin{gathered}
l^{-1}\left(\nu_{e}+J \rightarrow \bar{\nu}_{e}\right)=\frac{\left|g_{e e}\right|^{2}}{4 \pi} \frac{\mu_{\nu_{e}}}{q}\left|V_{e}\right|, \\
l^{-1}\left(\nu_{e}+J \rightarrow \bar{\nu}_{\beta}\right)=\frac{\left|g_{e \beta}\right|^{2}}{8 \pi} \frac{\left|V_{e}+V_{\beta}\right|}{q}\left(\mu_{\nu_{e}}-T \ln \left(\frac{e^{q / T}+1}{e^{q / T}}\right)\right), \\
l^{-1}\left(\nu_{\beta}+J \rightarrow \bar{\nu}_{\alpha}\right)=\frac{\left|g_{\beta \alpha}\right|^{2}}{8 \pi} \frac{1}{q}\left(\left|V_{\beta}+V_{\alpha}\right|\right) \frac{T e^{q / T}\left(q / T+\ln 2-\ln \left(e^{q / T}+1\right)\right.}{e^{q / T}-1}
\end{gathered}
$$

and

$$
l^{-1}\left(\nu_{\beta}+J \rightarrow \bar{\nu}_{e}\right)=\frac{\left|g_{\beta e}\right|^{2}}{8 \pi} \frac{0.7 T}{q}\left(\left|V_{\beta}+V_{e}\right|\right),
$$

where $q$ is the energy of $J$ and $\alpha$ and $\beta$ are either $\mu$ or $\tau$. The requirement $l^{-1}>R^{-1}$ implies that

$$
\begin{aligned}
& \left|g_{e e}\right| \gtrsim 6 \times 10^{-6}\left(\frac{q}{10 \mathrm{MeV}}\right)^{\frac{1}{2}}\left(\frac{200 \mathrm{MeV}}{\mu_{\nu_{e}}}\right)^{\frac{1}{2}}\left(\frac{0.3 \mathrm{eV}}{\left|V_{e}\right|}\right)^{\frac{1}{2}}, \\
& \left|g_{e \mu}\right|,\left|g_{e \tau}\right| \gtrsim 2 \times 10^{-6}\left(\frac{q}{10 \mathrm{MeV}}\right)^{\frac{1}{2}}\left(\frac{200 \mathrm{MeV}}{\mu_{\nu_{e}}}\right)^{\frac{1}{2}}\left(\frac{10 \mathrm{eV}}{\left|V_{\mu}\right|}\right)^{\frac{1}{2}}
\end{aligned}
$$

and

$$
\left|g_{\mu \mu}\right|,\left|g_{\tau \tau}\right|, \sqrt{2}\left|g_{\tau \mu}\right| \gtrsim 4 \times 10^{-6} .
$$

In the last case, the bound is derived for $q=10 \mathrm{MeV}, T=10 \mathrm{MeV}$ and $\left|V_{\mu}\right|=10 \mathrm{eV}$. Note that these bounds are derived for the parameters inside the inner core. In the outer core $\mu_{\nu_{e}}$ is much smaller and therefore the mean free path is much larger, i.e., in the outer core neutrinos can escape more easily. Apparently if the coupling constants are smaller than the bounds in Eqs. $(42,49,50)$, Majorons will leave the star core before undergoing any interaction. For $6\left|g_{\mu \mu}\right|\left(T / \mu_{\nu_{e}}\right)<\left|g_{e \mu}\right| \sim 2 \times 10^{-6}$, although the Majorons produced in the outer core escape immediately (recalling that the bound in Eq. (52) is extracted by studying the process $\bar{\nu}_{\mu} \rightarrow \nu_{e}+J$ in the outer core), the interaction of Majoron particles with $\nu_{e}$ in the inner core is not negligible.

For larger values of coupling, Majorons may become trapped or decay before leaving the star 
and the energy transfer by Majoron emission will become harder, but this does not mean that Majoron production does not affect the supernova evolution. To calculate the exact effect and to extract lower bounds on coupling constants, one needs to revisit the matter distribution and its time evolution including the effect of energy transfer by Majorons. That is beyond the scope of this paper. Here, we have discussed only the dominant interaction modes for larger values of the coupling constants. We recall that for $|g| \gtrsim 5 \times 10^{-4}$ the effective mass of the Majoron becomes non-negligible.

\section{Conclusions and discussions}

We have explored the energy loss from the inner core due to emission of Majorons. We have found that at early instants after the shock bounce $\left(t \lesssim 1\right.$ sec) when $V_{e}$ is positive, although the decay $\nu_{e} \rightarrow \bar{\nu}_{e}+J$ takes place, the period is too short to have significant energy transfer and therefore the energy loss due to $\nu_{e} \rightarrow \bar{\nu}_{e}+J$ does not imply any bound on $\left|g_{e e}\right|$. In the next period ( $t>1 \mathrm{sec}$ ) when $V_{e}<0$, neutrino decay is no longer kinematically allowed and instead the two processes $\bar{\nu}_{e} \rightarrow \nu_{e}+J$ and $\nu_{e}+\nu_{e} \rightarrow J$ can take place. Since the density of $\nu_{e}$ is much higher in the inner core, the process $\nu_{e}+\nu_{e} \rightarrow J$ implies a stronger bound. We have found that $\left|g_{e e}\right|<10^{-7}$ (see Eq. (42)) if the emitted Majoron leaves the core immediately after production. We have found that for $|g|<10^{-7}$ the effect of fourpoint processes $\left(\nu_{e}+\nu_{e} \rightarrow J+J\right.$ and $\left.\bar{\nu}_{e}+\nu_{e} \rightarrow J+J\right)$ is negligible. We believe that previous treatments of the reaction (e.g., [15]) have not correctly subtracted the 3-particle subprocesses.

We also have studied the bounds on coupling of Majoron to muon (tau) neutrino. In the basis in which $g_{\mu \tau}=0$ (since $\nu_{\mu}$ and $\nu_{\tau}$ are equivalent for supernova processes, we can always rotate $\left(\nu_{\tau}, \nu_{\mu}\right)$ to a new basis $\left(\nu_{\tau}^{\prime}, \nu_{\mu}^{\prime}\right)$ for which $\left.g_{\nu_{\tau}^{\prime} \nu_{\mu}^{\prime}}=0\right)$ we have found the following results. For $\left|g_{e \mu}\right|^{2} /\left|g_{\mu \mu}\right|^{2} \times \mu_{\nu_{e}}^{2} / T^{2}<37$, the processes $\bar{\nu}_{\mu} \rightarrow \nu_{\mu}+J$ and $\nu_{\mu}+\nu_{\mu} \rightarrow J$ imply $\left|g_{\mu \mu}\right|<8 \times 10^{-7}$ (see Eq. (49)) while $\nu_{e}+\nu_{\mu} \rightarrow J$ gives $\left|g_{e \mu}\right|<5 \times 10^{-7}$ (see Eq. (50)), providing the emitted Majoron leave the core without being trapped or undergoing decay. For $\left|g_{e \mu}\right|^{2} /\left|g_{\mu \mu}\right|^{2} \times\left(\mu_{\nu_{e}}^{2}\right) / T^{2}>37$, we have shown that the process $\nu_{e}+\nu_{\mu} \rightarrow J$ eats up $\nu_{\mu}$ within a short period (leading to a negative chemical potential for $\nu_{\mu}$ ) such that the bound from $\nu_{e}+\nu_{\mu} \rightarrow J$ no longer applies. However, in this case, the density of $\bar{\nu}_{\mu}$ increases $\left(\mu_{\bar{\nu}_{\mu}}=-\mu_{\nu_{\mu}}\right.$ becomes positive) and the bound on $\left|g_{\mu \mu}\right|$ (Eq. (49)) still applies (actually it will be a conservative one). For $\left|g_{e \mu}\right|>\left|g_{\mu \mu}\right| \sqrt{37} T / \mu_{\nu_{e}}$, the $\bar{\nu}_{\mu}$-decay in the outer core (where $\mu_{\nu_{e}} / T \lesssim 1$ ) imposes the strongest bound on $\left|g_{e \mu}\right|$ which is $\left|g_{e \mu}\right|<$ few $\times 10^{-6}$. These 
upper bounds are schematically summarized in Figs. (1) and (2). Note that the bounds on $\left|g_{\tau \tau}\right|$ and $\left|g_{e \tau}\right|$ are exactly the same as those on $\left|g_{\mu \mu}\right|$ and $\left|g_{e \mu}\right|$, respectively. The bounds are parameterized in terms of supernova parameters ( $T$, chemical potentials, $V$ and the radius of the core) so it is possible to apply the predictions of any supernova model.

All these upper bounds come from three-particle processes shown in diagrams $(a, b)$ of Fig. (2). In these processes, all the involved particles are on-shell. Therefore the aforementioned bounds can be translated into bounds on the corresponding element of the matrix $h$ in the derivative form of interaction (see Eq. (2)), using the relation given in Eq. (6).

We also studied Majoron decay and the interactions that can trap Majorons. We have found that the processes $\nu_{e}+J \rightarrow \bar{\nu}_{e}, \nu_{e}+J \rightarrow \bar{\nu}_{\mu, \tau}$ and $\nu_{\mu, \tau}+J \rightarrow \bar{\nu}_{\mu, \tau}$ may have significant effect $\left(l^{-1}>R_{\text {core }}^{-1}\right)$, only if $\left|g_{e e}\right|>6 \times 10^{-6}(q / 10 \mathrm{MeV})^{1 / 2},\left|g_{e \mu(\tau)}\right|>2 \times 10^{-6}(q / 10 \mathrm{MeV})^{1 / 2}$ and $\left|g_{\mu(\tau) \mu(\tau)}\right|>4 \times 10^{-6}$, respectively. If the couplings of Majorons to neutrinos are larger than these limits, the Majorons cannot leave the core immediately. However, the processes involving the Majoron still affect the evolution of supernova, transferring energy from the inner core and distorting the density distribution of the particles. If the couplings of Majoron are larger than some lower bounds, the only Majoron particles that can leave the core and cool down it are those produced in (or diffused into) a shell close to the neutrino-sphere where the density decreases rapidly with increasing radius. In this region the density is too low to give rise to a significant Majoron flux (i.e., $\mathcal{L}_{J} \ll \mathcal{L}_{\nu}$ ). We emphasize that to derive the lower bounds, it is not sufficient to consider the coupling constants collectively. For example, if $\left|g_{e \mu}\right|>5 \times 10^{-6}$, the Majorons produced via $\nu_{e}+\nu_{e} \rightarrow J$ can annihilate with another $\nu_{e}$ into $\bar{\nu}_{\mu}$ before escaping the core.

To derive the lower bounds, one must recalculate the density and temperature profiles of matter, neutrinos and Majoron particles which, in general, are different from those calculated so far without including Majoron processes. Here, we have discussed and evaluated only the four-point interactions which for large values of coupling constants may have significant effect.

\section{Acknowledgment}

I would like to thank J. Beacom, N. F. Bell, A. Burrows, H. Quinn and A. Yu. Smirnov for useful comments. I am also especially grateful to Michael Peskin for fruitful discussions and encouragement. 


\section{References}

[1] T. Toshito [SuperKamiokande Collaboration], hep-ex/0105023; S. Fukuda et. al., [SuperKamiokande Collaboration], Phys. Rev. Lett. 86 (2001) 5651; Phys. Rev. Lett. 86 (2001) 5656; S. fukuda et. al., [SuperKamiokande Collaboration], Phys. Lett. B. 539 (2002) 179; Q. R. Ahmad et. al., [SNO Collaboration], Phys. Rev. Lett. 87 (2001) 071301; Phys. Rev. Lett. 89 (2002) 011301; Phys. Rev. Lett. 89 (2002) 011302.

[2] J. Bonn et al., Nucl. Phys. Proc. Suppl. 91 (2001) 273; V. M. Lobashev et al., Proc. of the Int. Conf. Neutrino 2000, Sudbury, Canada, Nucl. Proc. Suppl. 77 (1999) 327; V. M. Lobashev et al., Nucl. Phys. Proc. Suppl. 91 (2001) 280; V. M. Lobashev, Prog. Part. Nucl. Phys. 48 (2002) 123.

[3] Y. Chikasige, R. Mohapatra and R. Peccei, Phys. Rev. Lett. 45 (1980) 1926.

[4] G. Gelmini and M. Roncadelli, Phys. Lett. B99 (1981) 411; H. M. Georgi, S. L. Glashow and S. Nussinov, Nucl. Phys. B193 (1981) 297; A. Yu. Smirnov, Yad. Fiz. 34 (1981) 1547; A. Santamaria and J. W. F. Valle, Phys. Lett. B195 (1987) 423; Phys. Rev. Lett. 60 (1988) 397; S. Bertolini and A. Santamaria, Nucl. Phys. B310 (1988) 714.

[5] D. S. P. Dearborn, D. N. Schramm and G. Steigman, Phys. Rev. Lett. 56 (1986) 26; M. Fukugita, S. Watamura and M. Yoshimura, Phys. Rev. Lett. 48 (1982) 1522 and Phys. Rev. D26 (1982) 1840; S. Bertolini and A. Santamaria, Phys. Lett. B220 (1989) 597.

[6] K. Choi and A. Santamaria, Phys. Rev. D42 (1990) 293.

[7] S. Pakvasa, hep-ph/0004077 and references therein.

[8] A. Bandyopadhyay, S. Choubey and S. Goswami, hep-ph/0204173, J. N. Bahcall, N. Cabbibo and A. Yahil, Phys. Lett. 28 (1972) 316; S. Pakvasa and K. Tennakone, Phys. Rev. Lett. 28 (1972) 1415; A. Acker, A. Joshipura and S. Pakvasa, Phys. Lett. B285 (1992) 371; S. Choubey, S. Goswami and D. Majumdar, Phys. Lett. B484 (2000) 73; A. Bandyopadhyay, S. Choubey and S. Goswami, Phys. Rev. D36 (2001) 113019; R. S. Raghavan, X. G. He and S. Pakvasa, Phys. Rev. D38 (1988) 1317; M. Lindner, T. Ohlsson and W. Winter, Nucl. Phys. B607 (2001) 326; D. Indumathi, hep-ph/0212038.

[9] V. Barger et. al., Phys. Rev. Lett. 82 (1999) 2640; V. Barger et. al., Phys. Lett. B462 (1999) 109; G. L. Fogli et. al., Phys. Rev. D59 (1999) 117303; P. Lipari and M. Lusignoli, 
Phys. Rev. D60 (1999) 013003; S. Choubey and S. Goswami, astropart. Phys. 14 (2000) 67.

[10] J. F. Beacom and N. F. Bell, Phys. Rev. D65 (2002) 113009.

[11] M. Lindner, T. Ohlsson and W. Winter, Nucl. Phys. B622 (2002) 429; J. A. Frieman, H. E. Haber and K. Freese, Phys. Lett. B200 (1988) 115; Y. Aharonov, F. T. Avignone and S. Nussinov, Phys. Lett. B200 (1988) 122; J. M. Soares and Wolfenstein, Phys. Rev. D40 (1989) 3666; A. Acker, S. Pakvasa and R. S. Raghavan, Phys. Lett. B238 (1990) 117.

[12] T. Bernatowicz et al., Phys. Rev. Lett. 69 (1992) 2341.

[13] D. I. Britton et al., Phys. Rev. D49 (1994) 28; V. Barger et al., Phys. Rev. D25 (1982) 907; G. B. Gelmini, S. Nussinov and M. Roncadelli, Nucl. Phys. B209 (1982) 157.

[14] Z. G. Berezhiani and A. Yu. Smirnov, Phys. Lett. B220 (1989) 279.

[15] M. Kachelriess, R. Tomas and J. W. F. Valle, Phys. Rev. D62 (2000) 023004.

[16] E. W. Kolb, D. L. Tubbs and D. A. Dicus, Astrophys. J. 255, L57 (1982); Nucl. Phys. B223 (1983) 826.

[17] G. M. Fuller, R. Mayle and J. R. Wilson, Astrophys. J. 332 (1988) 826.

[18] K. Sato and H. Suzuki, Phys. Lett. B196 (1987) 267; H.-T. Janka, in Proc. 5th Ringberg Workshop on Nuclear Astrophysics, Schloß Ringberg 1989.

[19] C. Giunti, C. W. Kim and U. V. Lee and W. P. Lam, Phys. Rev. D45 (1992) 1557.

[20] J. A. Grifols, E. Masso and S. Peris, Phys. Lett. B215 (1988) 593.

[21] R.V.Konoplich and M.Yu.Khlopov, Yadernaya Fizika 47 (1988) 891; Sov. J. Nucl. Phys. 47 (1988) 565.

[22] G. G. Raffelt, "Stars as Laboratories for Fundamental Physics", University of Chicago press, 1996.

[23] J. Pantaleone, Phys. Lett. B287 (1992) 128; Phys. Rev. D46 (1992) 510; A. Friedland and C. Lunardini, work in preparation.

[24] F. J. Botella, C. S. Lim and W. J. Marciano, Phys. Rev. D35 (1987) 896. 
[25] E. K. Akhmedov, C. Lunardini and A. Yu. Smirnov, Nucl. Phys. B643 (2002) 339.

[26] http://ik1au1.fzk.de/ katrin/

[27] S. W. Bruenn, Astrophys. J. 340 (1989) 955; E. Baron et. al., Phys. Rev. Lett. 59 (1989) 736.

[28] A. Burrows and J. M. Lattimer, Astrophys. J. 307 (1986) 178.

[29] W. Keil and H.-Th. Janka, Astron. Astrophys. 296 (1995) 145; J. A. Pons et. al., Astrophys. J. 513 (1999) 780.

[30] C. J. Horowitz and G. Li, Phys. Lett. B443 (1998) 58. 


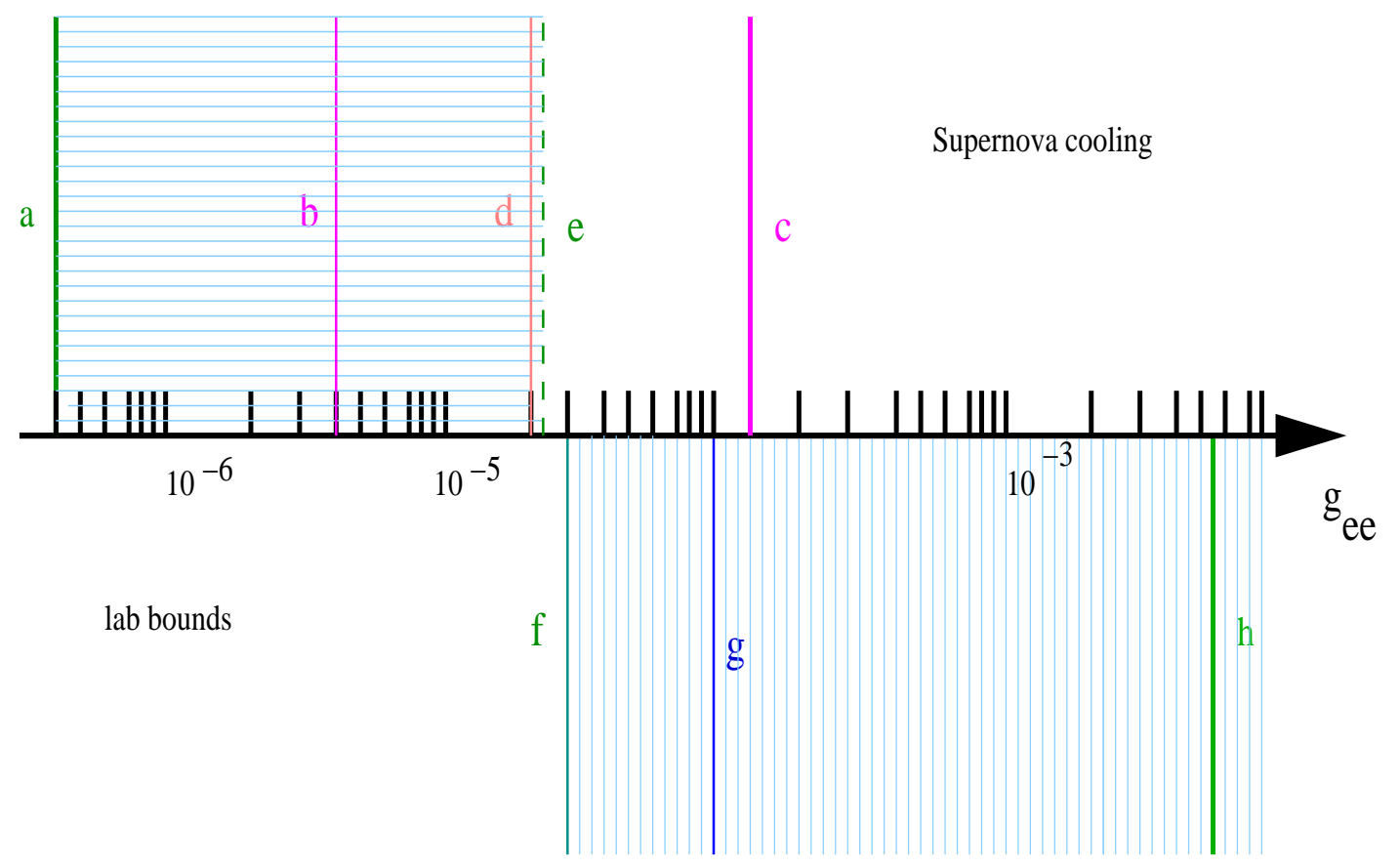

Figure 1: Bounds on $g_{e e}$ from supernova cooling (upper lines) and lab observations (lower lines). Line (a) shows the "upper" bound on $g_{e e}$ from the $\nu_{e} \nu_{e} \rightarrow J$ process in the supernova core (see Eq. (42)) while line (b) represents the "upper" bound from $\bar{\nu}_{e} \rightarrow \nu_{e} J$ [14]. Line (c) shows the "lower" bound which is derived without considering the effect of the four-point processes [14]. Line (c) gives the "lower" bound according to [15], we have argued that this is an overestimation. Thus we expect the true "lower" bound (e) to be between lines (c) and (d). The range of parameters between the "upper" and "lower" bounds (the horizontally shaded area) is excluded by supernova considerations. Line (f) represents the upper bound from double beta decay [12] and the whole region to its right (the vertically shaded area) is excluded. Lines $(\mathrm{g})$ and $(\mathrm{h})$ represent the upper bounds derived from solar neutrinos [10] and Kaon decay [13], respectively. Note that the bound (f) from solar data applies to $g_{21}$ rather than $g_{e e}$; we have included this line to compare the orders of magnitudes of different bounds. We have not resolved whether the "lower" bound (e) lies above or below (f); in the latter case, there is a small allowed region between the two bounds. 


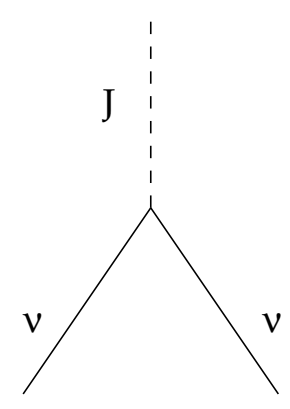

a)

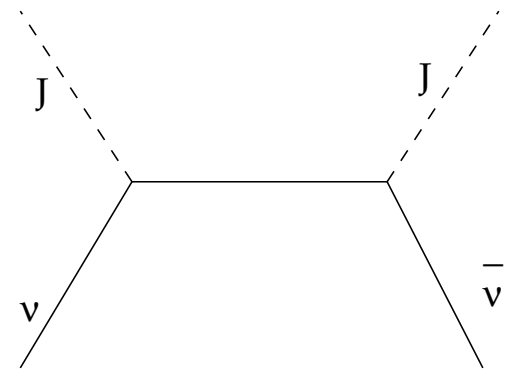

c)

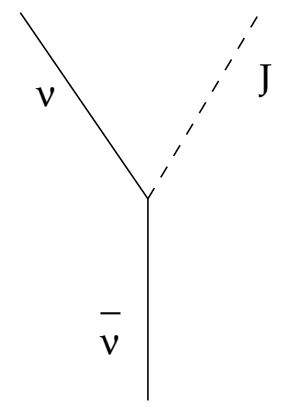

b)

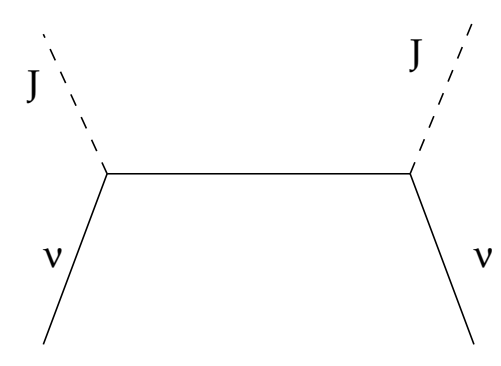

d)

Figure 2: Diagrams (a) and (b) are the dominant three-point processes and are possible only for $V<0$. Diagrams (c) and (d) are the subdominant diagrams and can take place for any value of $V$. 


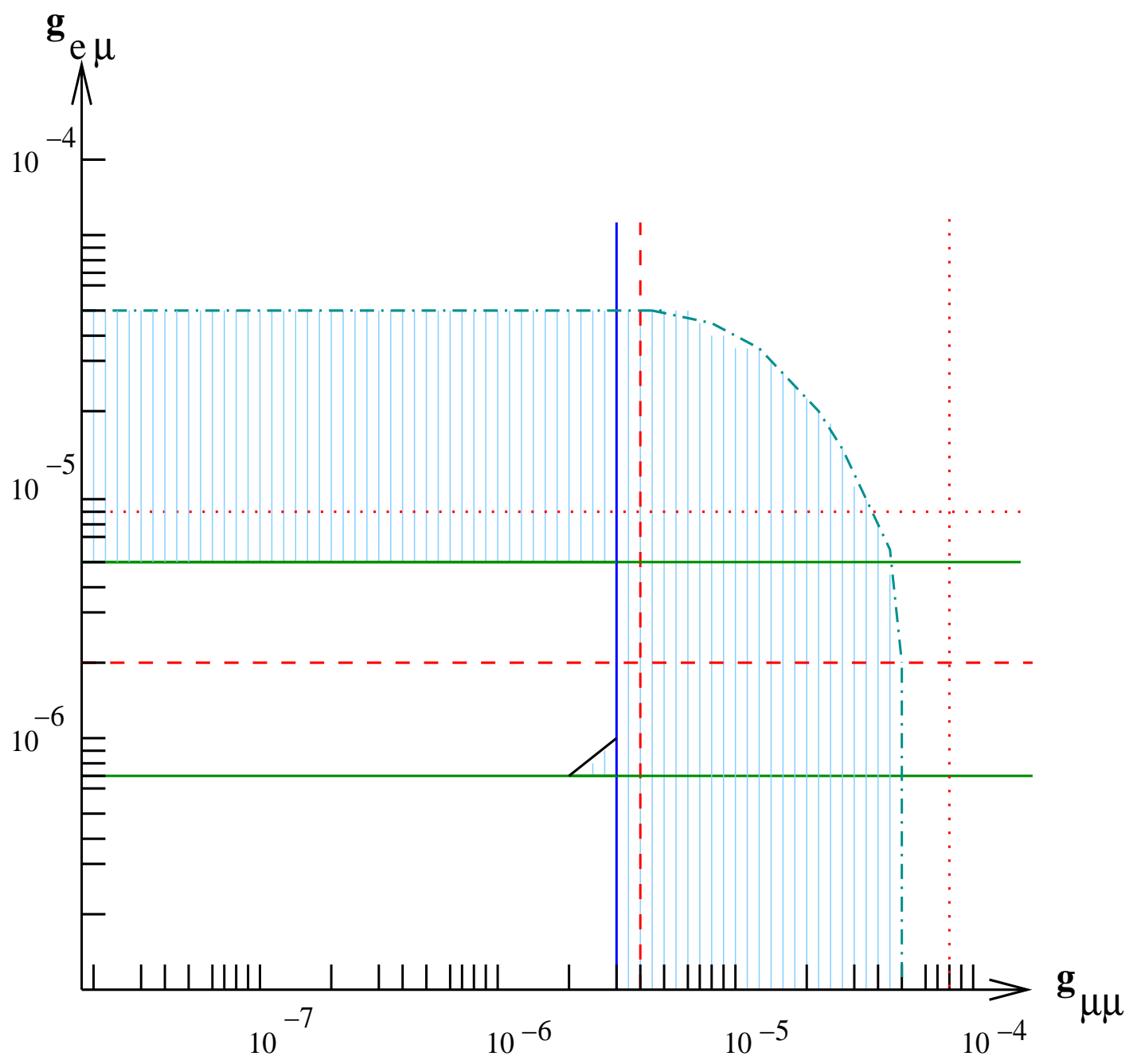

Figure 3: The bounds on coupling constants for $T=10 \mathrm{MeV}$ and $\mu_{\nu_{e}}=200 \mathrm{MeV}$. The shaded area is excluded by energy loss considerations. The horizontal and vertical lines at $5 \times 10^{-6}$ and $3 \times 10^{-6}$ represent the upper bounds obtained in Eq. (52) and Eq. (49), respectively. The dashed lines show the limits above which Majorons with energy $\sim 10 \mathrm{MeV}$ scatter before leaving the core. The dotted lines represent the same limits for Majorons with energy $\sim 200 \mathrm{MeV}$ (see Eqs. (62,63)). The dot-dashed line schematically represents the "lower" bound. We have not calculated the exact numerical value of the lower bound, but this is an estimate for $g_{e e}=0$. Note that the energies of Majorons produced via $\nu_{\mu} \nu_{\mu} \rightarrow J$ and $\bar{\nu}_{\mu} \rightarrow J \nu_{\mu}$ are of the order of $10 \mathrm{MeV}$; that is why the "lower" bound can be to the left of the vertical dotted line. The values inside the notch (above the horizontal line at $7 \times 10^{-7}$ ) are excluded due to $\nu_{\mu} \nu_{e} \rightarrow J$ (see Eq. (50) and its discussion). 


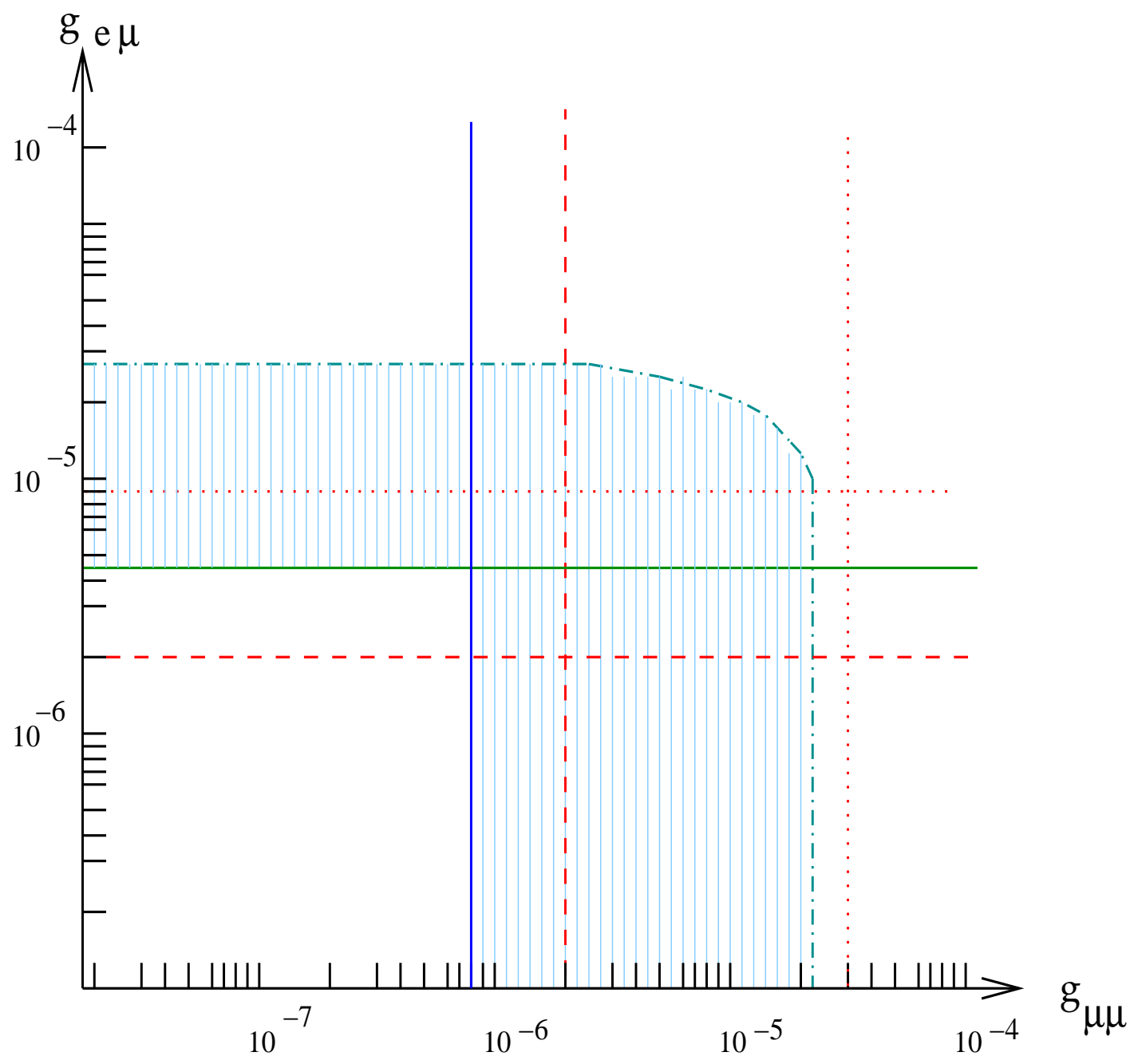

Figure 4: The bounds on coupling constants for $T=20 \mathrm{MeV}$ and $\mu_{\nu_{e}}=200 \mathrm{MeV}$. The shaded area is excluded by energy loss considerations. The horizontal and vertical lines at $4.5 \times 10^{-6}$ and $8 \times 10^{-7}$ represent the upper bounds obtained in Eq. (52) and Eq. (49), respectively. The dashed lines show the limits above which Majorons with energy $\sim 10 \mathrm{MeV}$ scatter before leaving the core. The dotted lines represent the same limits for Majorons with energy $\sim 200 \mathrm{MeV}$ (see Eqs. (62,63)). The dot-dashed line schematically represents the "lower" bound. We have not calculated the exact numerical value of the lower bound, but this is an estimate for $g_{e e}=0$. Note that the energies of Majorons produced via $\nu_{\mu} \nu_{\mu} \rightarrow J$ and $\bar{\nu}_{\mu} \rightarrow J \nu_{\mu}$ are of the order of $10 \mathrm{MeV}$; that is why the "lower" bound can be to the left of the vertical dotted line. 